\title{
A comprehensive analysis of the electric field distribution in an electrodynamic screen
}

\author{
Arash Sayyah, Mark N. Horenstein, and Malay K. Mazumder
}

\section{Abstract}

\section{Index Terms}

Self-cleaning, Electrostatics, Dust, Electrodynamic

\section{CONTENTS}

\begin{tabular}{|lrr}
\hline 1 & Introduction & 4
\end{tabular}

\begin{tabular}{|ll}
\hline 2 & Analytical Solution for the Electric Field
\end{tabular}

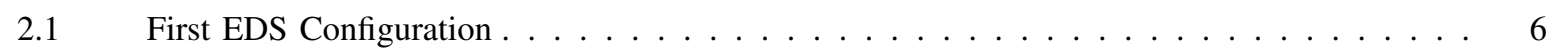

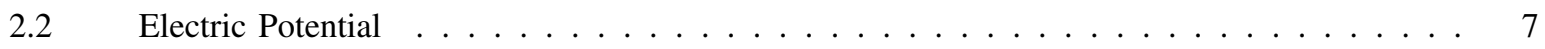

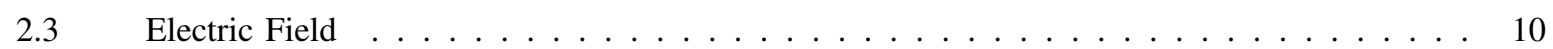

\begin{tabular}{|lll}
\hline 3 & Numerical Solution & 16
\end{tabular}

$3.1 \quad$ Verification of Analytical Approach $\ldots \ldots \ldots \ldots \ldots \ldots$

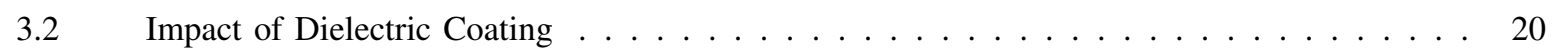

All the authors are with the Department of Electrical and Computer Engineering, Boston University, Boston, MA, 02215 USA e-mail: arashs@bu.edu Telephone: +1 217-979-7106 


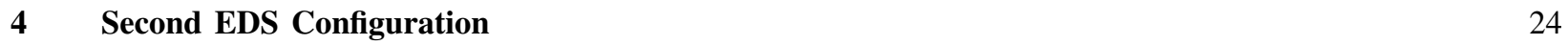

24

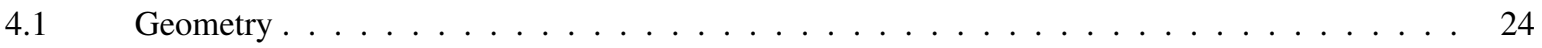

\section{LIST OF FIGURES}

30

1 Schematic of the EDS configuration with two stacked layers of transparent dielectric coatings. The solar

31

module could be a photovoltaic cell or a reflecting mirror film in a concentrated solar power application.

32

The dust particles, not depicted in the sketch, deposit on the dielectric layer 2. The electrodes are

connected to a three-phase power supply. The problem is assumed to be infinite in the $z$ direction

34 (normal to the page).

35

2 Numerical simulation results using finite element analysis software for the (a) electric field norm (|E $\mid$ )

36 and (b) electric potential of the EDS model in one fundamental spatial period $T_{s}$. The unit of the

37 vertical and horizontal axes is meter. The height of the electrodes have been considered to be $5 \mu \mathrm{m}$

$38 \quad$ and the maximum of the electric field norm reaches $3.2298 \times 10^{7} \mathrm{~V} / \mathrm{m}$. The voltages of phases A, B,

39 and C with Chromium electrodes are $1 \mathrm{kV}, 0$, and $1 \mathrm{kV}$, respectively. The electrode width and inter-

$40 \quad$ electrode spacing are considered to be $w=50 \mu \mathrm{m}$ and $g=200 \mu \mathrm{m}$, respectively. The thicknesses of

41 the first and second user-defined dielectric layers are $\delta_{1}=50 \mu \mathrm{m}$ and $\delta_{2}=100 \mu \mathrm{m}$, respectively, and

$42 \quad$ their relative permittivities are $\epsilon_{d 1}=5.14$ and $\epsilon_{d 2}=6.7 . \ldots \ldots \ldots \ldots \ldots \ldots$

${ }_{43} 3$ Three-phase rectangular voltage pulses with $50 \%$ duty cycle in one period $T_{t} . \ldots \ldots 18$ 
$4 \quad$ Comparison of analytical (ANA) results with numerical simulations using finite element analysis (FEA)

software for $w=g=100 \mu \mathrm{m}$ in one fundamental spatial period $T_{s}=600 \mu \mathrm{m}$ for six different voltage

combinations for phases A, B, and C. The magnitude of the electric field (unit: [MV/m]) on the EDS

surface (i.e. $y=\delta_{1}+\delta_{2}$ ) is illustrated vs. $x$. The positions of the first, second, and third electrodes are

at $[0,100 \mu \mathrm{m}],[200 \mu \mathrm{m}, 300 \mu \mathrm{m}]$, and [400 $\mu \mathrm{m}, 500 \mu \mathrm{m}]$, respectively. The voltages of phases (A,B,C)

for the cases (1) to (6) are respectively as follows: $(V, 0, V),(V, 0,0),(V, V, 0),(0, V, 0),(0, V, V)$, and

$(0,0, V)$, in which $V=1000$ volts. The thicknesses of the first and second dielectric coatings are 50

and $100 \mu \mathrm{m}$, respectively, and their relative permittivities are 5.14 and $6.7 . \ldots \ldots \ldots$

5 Comparison of the voltage profiles used in the analytical (ANA) approach and computed with numerical

simulations using finite element analysis (FEA) software for $w=g=100 \mu \mathrm{m}$ in one fundamental

spatial period $T_{s}=600 \mu \mathrm{m}$ for six different voltage combinations for phases A, B, and C. The electric

potential at $y=0$, where the electrodes are located is shown vs. $x$. The positions of the first, second,

and third electrodes are at $[0,100 \mu \mathrm{m}],[200 \mu \mathrm{m}, 300 \mu \mathrm{m}]$, and $[400 \mu \mathrm{m}, 500 \mu \mathrm{m}]$, respectively. The

rest of the parameters are identical to Fig. $4 \square \ldots \ldots \ldots \ldots \ldots \ldots \ldots \ldots \ldots \ldots$

6 The comparison between the $E_{y}$ (unit: [MV/m]) components on the EDS surface in one fundamental

spatial period for different values of the thickness of the first dielectric coating $\delta_{1}$. The results obtained

from the COMSOL Multiphysics software. The positions of the first, second, and third electrodes are

at $[0,50 \mu \mathrm{m}],[750 \mu \mathrm{m}, 800 \mu \mathrm{m}]$, and [1500 $\mu \mathrm{m}, 1550 \mu \mathrm{m}]$, respectively, and the fundamental spatial

period is $2250 \mu \mathrm{m}$. The voltages of phases (A,B,C) for the cases (1) to (6) are respectively as follows:

$(V, 0, V),(V, 0,0),(V, V, 0),(0, V, 0),(0, V, V)$, and $(0,0, V)$, in which $V=1000$ volts. The thickness of

the second dielectric coating is fixed at $100 \mu \mathrm{m}$ and the relative permittivities of the first and second

dielectric layers are 5.14 and 6.7 , respectively. $\ldots \ldots \ldots \ldots \ldots \ldots \ldots$

7 The second EDS configuration, in which the electrodes are printed on the lower side of dielectric layer

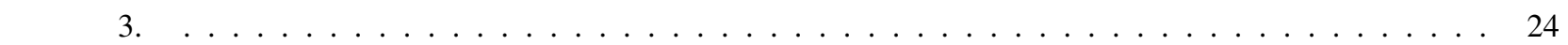




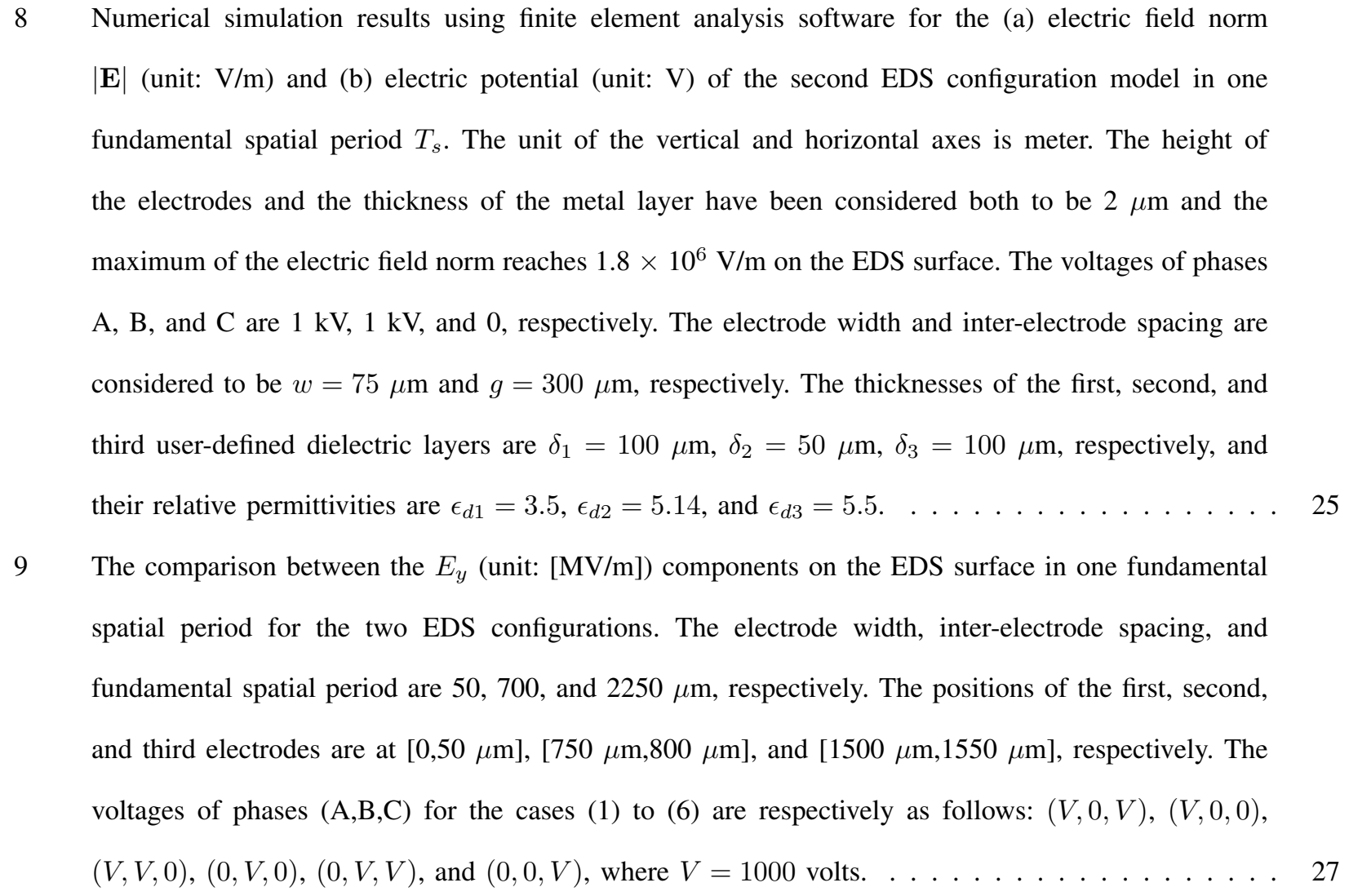

\section{INTRODUCTION}

Dust accumulation on the optical surfaces of solar energy harvesting devices such as flat-plate photovoltaic (PV) panels, concentrated solar mirrors, and concentrated photovoltaics (CPV) systems has an adverse impact on their performance by scattering and absorption of the incident sunlight. A literature survey on the dust accumulation problem by Sayyah et al [1] has shown that dust deposition can cause up to $1 \%$ loss in the daily output power in flat-plate PV installations located in arid and semi-arid areas of the world, where the average of annual solar 
and also is labor-intensive as it requires a team of trained personnel to perform the cleaning task. Furthermore, water cleaning use is not scalable, i.e., lack of it will impede growth of CSP and PV plants. Recently, there have been some advancements in utilizing robotic devices in cleaning flat-plate PV panels [5] yet they are still in the developmental stage, require water resources for cleaning, and their scalability in large-scale solar plants has not been well established.

In order to cope with the shortcomings of commonly-practiced cleaning methods, the concept of automatic dust removal using electrostatic forces is in developmental stages and on a commercialization path. The general idea of automatic dust removal was initially introduced by Tatom et al. [6] at NASA and then developed by Masuda $e t$ al. [7] [8] at the University of Tokyo in the 1970s for transporting charged aerosol particles. Although the concept has found a niche application for dust removal on solar modules to be used in Mars exploration missions [9], [10], [11], [12], there have been significant endeavors to adopt this technology to mitigate dust accumulation losses in solar energy harvesting plants [13], [14]. Electrodynamic screen (EDS) technology represents a viable promising solution as it does not require water resources or mechanical movement in removing dust particles, and it is an extremely low-power technology that can be fed from the harvesting device itself and does not need external power source. The EDS consists of inter-digitated electrodes deposited on a glass substrate, encapsulated by one or more transparent dielectric coating(s) that protect the electrodes from direct exposure to the outdoor environment. The EDS can be easily integrated onto a PV panel or the reflecting mirror in concentrated solar power (CSP) applications. The electrodes are connected to a single-phase or a multi-phase power supply that energizes the electrodes with a specified waveform pattern and frequency. Once the deposited dust particles are charged sufficiently, they are removed by the Coulomb force from the EDS surface.

Electric field distribution model plays a decisive role in performance evaluation of the EDS and how to increase its dust removal efficiency by manipulating the design parameters as well as material selection. This paper deals with the analysis of electric field distributions in two EDS configurations. The organization of this paper is as follows: the analytical solutions for the electric potential and electric field components for the first EDS configuration are presented in Section 2. The corroboration of analytical solutions has been addressed in Section 3. Section 4 provides the simulation results for the electric field distribution in the second EDS configuration and a comparison between two configurations. Finally, Section 5 provides the conclusions of this paper. 
\{seg:analyticalef \{fig:eds $\}$

\section{Analytical Solution for the Electric Field}

In this section, we provide closed-form solutions for the electric field and electric potential in the first EDS configuration. The analytical solutions show explicitly how various design parameters play role in the electric field distribution and provides valuable insight through the physical model of the problem. The solutions can be easily implemented for the dust particle trajectory studies.

\section{A. First EDS Configuration}

Figure 1 depicts the configuration of the EDS in the $x y$-plane. The electrodes are deposited on a glass substrate while their height is neglected in the calculations. In Fig. 11 the width of the electrodes and inter-electrode spacing are denoted as $w$ and $g$, respectively. The electrodes are encapsulated by two layers of transparent dielectric materials 1 and 2, with relative permittivities of $\epsilon_{d 1}$ and $\epsilon_{d 2}$, respectively. As an example in the practice, dielectric 1 is optically clear adhesive (OCA) and dielectric 2 is a very thin borosilicate glass. The electrodes have the same size and inter-electrode spacing and are connected to a three-phase power supply that provides traveling-waves to repel and convey dust particles. As the output of the power supply for energizing the electrodes is known at any given time $t$, regardless of the sinusoidal, rectangular, saw tooth, etc., the electrodes' voltages are known at each time instant and are represented as a function of time.

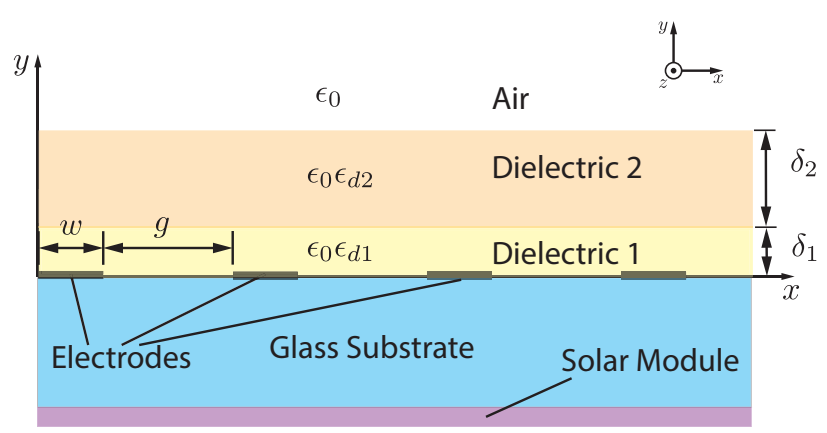

Fig. 1. Schematic of the EDS configuration with two stacked layers of transparent dielectric coatings. The solar module could be a photovoltaic cell or a reflecting mirror film in a concentrated solar power application. The dust particles, not depicted in the sketch, deposit on the dielectric layer 2. The electrodes are connected to a three-phase power supply. The problem is assumed to be infinite in the $z$ direction (normal to the page).

Since the electrodes are connected to a three-phase power supply, the fundamental spatial period, denoted as $T_{s}$, is expressed as

$$
T_{s}=3(w+g) .
$$


We assume that the electric field distribution is not affected by the charge of deposited dust particles on the EDS surface, i.e., they carry no net charge. Hence, in the absence of net electric charge in the region above the EDS surface, we can write Laplace's equation for the electric potential in the air, denoted as $\phi_{a}(x, y)$ as follows:

$$
\frac{\partial \phi_{a}^{2}(x, y)}{\partial x^{2}}+\frac{\partial \phi_{a}^{2}(x, y)}{\partial y^{2}}=0, \quad 0 \leq x \leq T_{s}, \quad \delta_{1}+\delta_{2} \leq y<\infty
$$

We have added the subscript " $a$ " that highlights the electric potential in the air. Similarly, the subscripts " $d 1$ " and " $d 2$ " denote the potentials in the dielectric layers 1 and 2 , respectively. The goal is to find the electric potential and the electric field components in the two layers of the dielectric materials and air. The electric field components in the air are the ones exerted on the dust particles and effective in the dust removal process. However, the electric field solutions within two dielectric layers can be used for further analysis of electric stress and dielectric breakdown.

\section{B. Electric Potential}

The electric potentials at $y=0, y=\delta_{1}$, and $y=\delta_{1}+\delta_{2}$ (air/dielectric 2 boundary) in Fig. 1 are functions of $x$ and are respectively expressed as:

$$
\begin{aligned}
v_{m}(x) & =\sum_{k=0}^{\infty} a_{k} \cos \left(k \Omega_{0} x\right)+b_{k} \sin \left(k \Omega_{0} x\right), \\
u_{m}(x) & =\sum_{k=0}^{\infty} g_{k} \cos \left(k \Omega_{0} x\right)+h_{k} \sin \left(k \Omega_{0} x\right), \\
w_{m}(x) & =\sum_{k=0}^{\infty} p_{k} \cos \left(k \Omega_{0} x\right)+q_{k} \sin \left(k \Omega_{0} x\right),
\end{aligned}
$$

where $a_{k}, b_{k}, g_{k}, h_{k}, p_{k}$, and $q_{k}$ are the Fourier coefficients, and $\Omega_{0}=\frac{2 \pi}{T_{s}}$. The Fourier coefficients $a_{k}$ and $b_{k}$ are known at each time instant as the electric potential applied to the electrodes is known, and $g_{k}, h_{k}, p_{k}$, and $q_{k}$ are unknown Fourier coefficients that need to be calculated. We can assume that the electric potential varies linearly between adjacent electrodes at $y=0$. Because applied voltages are time varying, the introduced Fourier coefficients are also functions of time; for instance $a_{k}(t)$ and $b_{k}(t)$. For the ease of representation, however, we drop the dependency upon " $t$ ". We may write the electric potential inside the dielectric materials in such a way that both boundary conditions at their boundaries are satisfied. We assume the potential inside the first dielectric is a summation of two functions, one a function of $v_{m}$ and the other a function of $u_{m}$, as defined in Eqs. 3 and 4 respectively: 


$$
\phi_{d 1}(x, y)=f_{1}\left(v_{m}\right)+f_{2}\left(u_{m}\right), \quad 0 \leq x \leq T_{s}, \quad 0 \leq y \leq \delta_{1}
$$

in which the functions $f_{1}$ and $f_{2}$ are variables of both $x$ and $y$. The function $f_{1}\left(v_{m}\right)$ is written as:

$$
f_{1}\left(v_{m}\right)=v_{m}\left(A_{1} e^{-k \Omega_{0} y}+A_{2} e^{k \Omega_{0} y}\right)
$$

$$
\begin{aligned}
& A_{1}=\frac{1}{2} e^{k \Omega_{0} \delta_{1}} \operatorname{csch}\left(k \Omega_{0} \delta_{1}\right) \\
& A_{2}=-\frac{1}{2} e^{-k \Omega_{0} \delta_{1}} \operatorname{csch}\left(k \Omega_{0} \delta_{1}\right)
\end{aligned}
$$

By looking into coefficients $A_{1}$ and $A_{2}$ obtained for $f_{1}\left(v_{m}\right)$, we can write the function $f_{2}\left(u_{m}\right)$ as:

$$
f_{2}\left(u_{m}\right)=u_{m} \frac{e^{k \Omega_{0} y}-e^{-k \Omega_{0} y}}{e^{k \Omega_{0} \delta_{1}}-e^{-k \Omega_{0} \delta_{1}}}
$$

in which $f_{2}\left(u_{m}\right)=0$ if $y=0$ and $f_{2}\left(u_{m}\right)=u_{m}$ at $y=\delta_{1}$. Therefore,

$$
f_{2}\left(u_{m}\right)=u_{m} \frac{\sinh \left(k \Omega_{0} y\right)}{\sinh \left(k \Omega_{0} \delta_{1}\right)}
$$

Using the expressions obtained for $f_{1}\left(v_{m}\right)$ and $f_{2}\left(u_{m}\right)$, the electric potential inside the first dielectric layer is expressed as:

$$
\phi_{d 1}(x, y)=-v_{m} \frac{\sinh \left[k \Omega_{0}\left(y-\delta_{1}\right)\right]}{\sinh \left(k \Omega_{0} \delta_{1}\right)}+u_{m} \frac{\sinh \left(k \Omega_{0} y\right)}{\sinh \left(k \Omega_{0} \delta_{1}\right)}, \quad 0 \leq x \leq T_{s}, \quad 0 \leq y \leq \delta_{1}
$$

\footnotetext{
Substitution of the Fourier series representation introduced for the $v_{m}$ and $u_{m}$ in Eqs. 3 and 4, respectively,
} 
yields the electric potential in the first dielectric material:

$$
\begin{aligned}
\phi_{d 1}(x, y)= & -\sum_{k} \frac{\sinh \left[k \Omega_{0}\left(y-\delta_{1}\right)\right]}{\sinh \left(k \Omega_{0} \delta_{1}\right)}\left[a_{k} \cos \left(k \Omega_{0} x\right)+b_{k} \sin \left(k \Omega_{0} x\right)\right] \\
& +\sum_{k} \frac{\sinh \left(k \Omega_{0} y\right)}{\sinh \left(k \Omega_{0} \delta_{1}\right)}\left[g_{k} \cos \left(k \Omega_{0} x\right)+h_{k} \sin \left(k \Omega_{0} x\right)\right], \quad 0 \leq x \leq T_{s}, \quad 0 \leq y \leq \delta_{1}
\end{aligned}
$$

A similar approach can be applied to the second dielectric layer, yielding the electric potential in the latter:

$$
\phi_{d 2}(x, y)=f_{3}\left(u_{m}\right)+f_{4}\left(w_{m}\right), \quad 0 \leq x \leq T_{s}, \quad \delta_{1} \leq y \leq \delta_{1}+\delta_{2}
$$

in which the functions $f_{3}$ and $f_{4}$ are variables of both $x$ and $y$. The function $f_{3}\left(u_{m}\right)$ is expressed as

$$
f_{3}\left(u_{m}\right)=u_{m}\left(A_{3} e^{-k \Omega_{0} y}+A_{4} e^{k \Omega_{0} y}\right)
$$

where the two constants $A_{3}$ and $A_{4}$ are unknown. The two boundary conditions in the second dielectric layer impose $f_{3}\left(u_{m}\right)=u_{m}$ and $f_{3}\left(u_{m}\right)=0$ at $y=\delta_{1}$ and $y=\delta_{1}+\delta_{2}$, respectively. Hence,

$$
\begin{cases}A_{3} e^{-k \Omega_{0} \delta_{1}}+A_{4} e^{k \Omega_{0} \delta_{1}}=1, & \text { if } y=\delta_{1} \\ A_{3} e^{-k \Omega_{0}\left(\delta_{1}+\delta_{2}\right)}+A_{4} e^{k \Omega_{0}\left(\delta_{1}+\delta_{2}\right)}=0 & \text { if } y=\delta_{1}+\delta_{2}\end{cases}
$$

The constants $A_{3}$ and $A_{4}$ are the solution set for the Eqs. 17

$$
\begin{aligned}
& A_{3}=\frac{e^{k \Omega_{0}\left(\delta_{1}+\delta_{2}\right)}}{e^{k \Omega_{0} \delta_{2}}-e^{-k \Omega_{0} \delta_{2}}}, \\
& A_{4}=-\frac{e^{-k \Omega_{0}\left(\delta_{1}+\delta_{2}\right)}}{e^{k \Omega_{0} \delta_{2}}-e^{-k \Omega_{0} \delta_{2}}} .
\end{aligned}
$$

For the second term in Eq. 15, i.e. $f_{4}\left(w_{m}\right)$, we would like to have $f_{4}\left(w_{m}\right)=0$ and $f_{4}\left(w_{m}\right)=w_{m}$ for $y=\delta_{1}$ and $y=\delta_{1}+\delta_{2}$, respectively. Hence, we can write $f_{4}\left(w_{m}\right)$ as

$$
f_{4}\left(w_{m}\right)=w_{m} \frac{e^{k \Omega_{0}\left(y-\delta_{1}\right)}-e^{-k \Omega_{0}\left(y-\delta_{1}\right)}}{e^{k \Omega_{0} \delta_{2}}-e^{-k \Omega_{0} \delta_{2}}}
$$




$$
\phi_{d 2}(x, y)=-u_{m} \frac{\sinh \left[k \Omega_{0}\left(y-\left(\delta_{1}+\delta_{2}\right)\right)\right]}{\sinh \left(k \Omega_{0} \delta_{2}\right)}+w_{m} \frac{\sinh \left[k \Omega_{0}\left(y-\delta_{1}\right)\right]}{\sinh \left(k \Omega_{0} \delta_{2}\right)}
$$

Using the expression introduced for the $u_{m}$ and $w_{m}$ in Eqs. 4 and 5 , the electric potential in the second dielectric layer is

$$
\begin{aligned}
\phi_{d 2}(x, y)= & -\sum_{k} \frac{\sinh \left[k \Omega_{0}\left[y-\left(\delta_{1}+\delta_{2}\right)\right]\right]}{\sinh \left(k \Omega_{0} \delta_{2}\right)}\left[g_{k} \cos \left(k \Omega_{0} x\right)+h_{k} \sin \left(k \Omega_{0} x\right)\right]+ \\
& \sum_{k} \frac{\sinh \left[k \Omega_{0}\left(y-\delta_{1}\right)\right]}{\sinh \left(k \Omega_{0} \delta_{2}\right)}\left[p_{k} \cos \left(k \Omega_{0} x\right)+q_{k} \sin \left(k \Omega_{0} x\right)\right] \quad 0 \leq x \leq T_{s}, \quad \delta_{1} \leq y \leq \delta_{1}+\delta_{2}
\end{aligned}
$$

Horenstein et al. [15] have provided the electric field solution for an EDS with no dielectric coating by solving Laplace's equation, where the deposited electrodes are exposed to the air. The electrodes are always coated so as to be protected from outdoor exposure. However, their solution can be adopted herein as a representation of the electric field in the air. The solution for the electric potential for the current configuration as in Fig. 1 1 is written as

$$
\phi_{a}(x, y)=\sum_{k} e^{-k \Omega_{0}\left[y-\left(\delta_{1}+\delta_{2}\right)\right]}\left[p_{k} \cos \left(k \Omega_{0} x\right)+q_{k} \sin \left(k \Omega_{0} x\right)\right], \quad 0 \leq x \leq T_{s}, \quad \delta_{1}+\delta_{2} \leq y<\infty
$$

It should be noted that the electric field potential in the air satisfies the following boundary conditions:

1) At $y=\delta_{1}+\delta_{2}: \phi_{a}\left(x, \delta_{1}+\delta_{2}\right)=\sum_{k}\left[p_{k} \cos \left(k \Omega_{0} x\right)+q_{k} \sin \left(k \Omega_{0} x\right)\right]=w_{m}$

2) When $y \rightarrow \infty: \phi_{a}(x, y)=0$

The electric potentials in the dielectric 1, dielectric 2, and air are obtained via the Eqs. 14,22 and 23 respectively.

\section{Electric Field}

Because we are able to write the electric potentials in the three dielectric media, the electric field components can also be calculated as follows. For the dielectric layer 1, the electric field $\mathbf{E}_{d 1}(x, y)$ is expressed as:

$$
\mathbf{E}_{d 1}(x, y)=E_{d 1, x} \mathbf{a}_{x}+E_{d 1, y} \mathbf{a}_{y}
$$

in which $E_{d 1, x}$ and $E_{d 1, y}$ are the electric field components in the $x$ and $y$ directions, respectively. The $\mathbf{a}_{x}$ and $\mathbf{a}_{y}$ are the unit vectors in the $x$ and $y$ directions, respectively. Similarly, for the electric fields in the dielectric layer 2 and air denoted as $\mathbf{E}_{d 2}$ and $\mathbf{E}_{a}$, respectively, we have: 


$$
\mathbf{E}_{d 2}(x, y)=E_{d 2, x} \mathbf{a}_{x}+E_{d 2, y} \mathbf{a}_{y},
$$

and

$$
\mathbf{E}_{a}(x, y)=E_{a, x} \mathbf{a}_{x}+E_{a, y} \mathbf{a}_{y} .
$$

Given that $\mathbf{E}=-\nabla \phi$, the electric potential (Eq. 14) results in electric field components of:

$$
\begin{aligned}
E_{d 1, x}= & -\frac{\partial \phi_{d 1}(x, y)}{\partial x} \\
= & \Omega_{0} \sum_{k} \frac{\sinh \left[k \Omega_{0}\left(y-\delta_{1}\right)\right]}{\sinh \left(k \Omega_{0} \delta_{1}\right)}\left[-k a_{k} \sin \left(k \Omega_{0} x\right)+k b_{k} \cos \left(k \Omega_{0} x\right)\right] \\
& -\Omega_{0} \sum_{k} \frac{\sinh \left(k \Omega_{0} y\right)}{\sinh \left(k \Omega_{0} \delta_{1}\right)}\left[-k g_{k} \sin \left(k \Omega_{0} x\right)+k h_{k} \cos \left(k \Omega_{0} x\right)\right]
\end{aligned}
$$

195

and

$$
\begin{aligned}
E_{d 1, y}= & -\frac{\partial \phi_{d 1}(x, y)}{\partial y} \\
= & \Omega_{0} \sum_{k} \frac{\cosh \left[k \Omega_{0}\left(y-\delta_{1}\right)\right]}{\sinh \left(k \Omega_{0} \delta_{1}\right)}\left[k a_{k} \cos \left(k \Omega_{0} x\right)+k b_{k} \sin \left(k \Omega_{0} x\right)\right] \\
& -\Omega_{0} \sum_{k} \frac{\left.\cosh \left(k \Omega_{0} y\right)\right]}{\sinh \left(k \Omega_{0} \delta_{1}\right)}\left[k g_{k} \cos \left(k \Omega_{0} x\right)+k h_{k} \sin \left(k \Omega_{0} x\right)\right]
\end{aligned}
$$

Based on the potential in the dielectric layer 2 expressed in Eq. 22, the $x$ and $y$ electric field components are:

$$
\begin{aligned}
E_{d 2, x}= & -\frac{\partial \phi_{d 2}(x, y)}{\partial x} \\
= & \Omega_{0} \sum_{k} \frac{\sinh \left[k \Omega_{0}\left[y-\left(\delta_{1}+\delta_{2}\right)\right]\right]}{\sinh \left(k \Omega_{0} \delta_{2}\right)}\left[-k g_{k} \sin \left(k \Omega_{0} x\right)+k h_{k} \cos \left(k \Omega_{0} x\right)\right] \\
& -\Omega_{0} \sum_{k} \frac{\sinh \left[k \Omega_{0}\left(y-\delta_{1}\right)\right]}{\sinh \left(k \Omega_{0} \delta_{2}\right)}\left[-k p_{k} \sin \left(k \Omega_{0} x\right)+k q_{k} \cos \left(k \Omega_{0} x\right)\right]
\end{aligned}
$$




$$
\begin{aligned}
E_{d 2, y}= & -\frac{\partial \phi_{d 2}(x, y)}{\partial y} \\
= & \Omega_{0} \sum_{k} \frac{\cosh \left[k \Omega_{0}\left[y-\left(\delta_{1}+\delta_{2}\right)\right]\right]}{\sinh \left(k \Omega_{0} \delta_{2}\right)}\left[k g_{k} \cos \left(k \Omega_{0} x\right)+k h_{k} \sin \left(k \Omega_{0} x\right)\right] \\
& -\Omega_{0} \sum_{k} \frac{\cosh \left[k \Omega_{0}\left(y-\delta_{1}\right)\right]}{\sinh \left(k \Omega_{0} \delta_{2}\right)}\left[k p_{k} \cos \left(k \Omega_{0} x\right)+k q_{k} \sin \left(k \Omega_{0} x\right)\right] .
\end{aligned}
$$

Electric field in the air is calculated based on the electric potential in Eq. 23.

$$
\begin{aligned}
E_{a, x} & =-\frac{\partial \phi_{a}(x, y)}{\partial x} \\
& =\Omega_{0} \sum_{k} e^{-k \Omega_{0}\left[y-\left(\delta_{1}+\delta_{2}\right)\right]}\left[k p_{k} \sin \left(k \Omega_{0} x\right)-k q_{k} \cos \left(k \Omega_{0} x\right)\right]
\end{aligned}
$$

and

$$
\begin{aligned}
E_{a, y} & =-\frac{\partial \phi_{a}(x, y)}{\partial y} \\
& =\Omega_{0} \sum_{k} e^{-k \Omega_{0}\left[y-\left(\delta_{1}+\delta_{2}\right)\right]}\left[k p_{k} \cos \left(k \Omega_{0} x\right)+k q_{k} \sin \left(k \Omega_{0} x\right)\right] .
\end{aligned}
$$

$$
\begin{aligned}
E_{d 1, x}\left(x, \delta_{1}\right)= & \Omega_{0} \sum_{k}\left[k g_{k} \sin \left(k \Omega_{0} x\right)-k h_{k} \cos \left(k \Omega_{0} x\right)\right] \\
E_{d 1, y}\left(x, \delta_{1}\right)= & \Omega_{0} \sum_{k} \operatorname{csch}\left(k \Omega_{0} \delta_{1}\right)\left[k a_{k} \cos \left(k \Omega_{0} x\right)+k b_{k} \sin \left(k \Omega_{0} x\right)\right] \\
& -\Omega_{0} \sum_{k} \operatorname{coth}\left(k \Omega_{0} \delta_{1}\right)\left[k g_{k} \cos \left(k \Omega_{0} x\right)+k h_{k} \sin \left(k \Omega_{0} x\right)\right]
\end{aligned}
$$

At $y=\delta_{1}$, the electric field components for the second dielectric are: 


$$
\begin{aligned}
E_{d 2, x}\left(x, \delta_{1}\right)= & \Omega_{0} \sum_{k}\left[k g_{k} \sin \left(k \Omega_{0} x\right)-k h_{k} \cos \left(k \Omega_{0} x\right)\right] \\
E_{d 2, y}\left(x, \delta_{1}\right)= & \Omega_{0} \sum_{k} \operatorname{coth}\left[k \Omega_{0} \delta_{2}\right]\left[k g_{k} \cos \left(k \Omega_{0} x\right)+k h_{k} \sin \left(k \Omega_{0} x\right)\right] \\
& -\Omega_{0} \sum_{k} \operatorname{csch}\left(k \Omega_{0} \delta_{2}\right)\left[k p_{k} \cos \left(k \Omega_{0} x\right)+k q_{k} \sin \left(k \Omega_{0} x\right)\right]
\end{aligned}
$$

$$
\begin{aligned}
E_{d 2, x}\left(x, \delta_{1}+\delta_{2}\right)= & \Omega_{0} \sum_{k}\left[k p_{k} \sin \left(k \Omega_{0} x\right)-k q_{k} \cos \left(k \Omega_{0} x\right)\right] \\
E_{d 2, y}\left(x, \delta_{1}+\delta_{2}\right)= & \Omega_{0} \sum_{k} \operatorname{csch}\left(k \Omega_{0} \delta_{2}\right)\left[k g_{k} \cos \left(k \Omega_{0} x\right)+k h_{k} \sin \left(k \Omega_{0} x\right)\right] \\
& -\Omega_{0} \sum_{k} \operatorname{coth}\left(k \Omega_{0} \delta_{2}\right)\left[k p_{k} \cos \left(k \Omega_{0} x\right)+k q_{k} \sin \left(k \Omega_{0} x\right)\right]
\end{aligned}
$$

$$
\begin{aligned}
& E_{a, x}\left(x, \delta_{1}+\delta_{2}\right)=\Omega_{0} \sum_{k}\left[k p_{k} \sin \left(k \Omega_{0} x\right)-k q_{k} \cos \left(k \Omega_{0} x\right)\right], \\
& E_{a, y}\left(x, \delta_{1}+\delta_{2}\right)=\Omega_{0} \sum_{k}\left[k p_{k} \cos \left(k \Omega_{0} x\right)+k q_{k} \sin \left(k \Omega_{0} x\right)\right] .
\end{aligned}
$$

At this point, since the electric field components are available, we can match the boundary conditions at the two boundaries $y=\delta_{1}$ and $y=\delta_{1}+\delta_{2}$. At $y=\delta_{1}$, the tangential components must satisfy $E_{d 1, x}=E_{d 2, x}$. By looking into the expressions provided for $E_{d 1, x}$ and $E_{d 2, x}$ in Eqs. 33 and 35 , respectively, it is clear that the boundary condition for the tangential components is automatically satisfied. The boundary condition for the normal components at $y=\delta_{1}$ must satisfy $\epsilon_{0} \epsilon_{d 1} E_{d 1, y}=\epsilon_{0} \epsilon_{d 2} E_{d 2, y}$. Therefore,

$$
\begin{aligned}
& \left.\epsilon_{d 1}\left\{\sum_{k} \operatorname{csch}\left(k \Omega_{0} \delta_{1}\right)\left[k a_{k} \cos \left(k \Omega_{0} x\right)+k b_{k} \sin \left(k \Omega_{0} x\right)\right]-\sum_{k} \operatorname{coth}\left(k \Omega_{0} \delta_{1}\right)\left[k g_{k} \cos \left(k \Omega_{0} x\right)\right]+k h_{k} \sin \left(k \Omega_{0} x\right)\right]\right\}= \\
& \epsilon_{d 2}\left\{\sum_{k} \operatorname{coth}\left(k \Omega_{0} \delta_{2}\right)\left[k g_{k} \cos \left(k \Omega_{0} x\right)+k h_{k} \sin \left(k \Omega_{0} x\right)\right]-\sum_{k} \operatorname{csch}\left(k \Omega_{0} \delta_{2}\right)\left[k p_{k} \cos \left(k \Omega_{0} x\right)+k q_{k} \sin \left(k \Omega_{0} x\right)\right]\right\}(41)
\end{aligned}
$$

Re-arranging the expressions for the $\cos (\cdot)$ and $\sin (\cdot)$ terms in Eq. 41, we have: 


$$
\begin{aligned}
& \epsilon_{d 1}\left\{\sum_{k}\left[\operatorname{csch}\left(k \Omega_{0} \delta_{1}\right) k a_{k}-\operatorname{coth}\left(k \Omega_{0} \delta_{1}\right) k g_{k}\right] \cos \left(k \Omega_{0} x\right)+\left[\operatorname{csch}\left(k \Omega_{0} \delta_{1}\right) k b_{k}-\operatorname{coth}\left(k \Omega_{0} \delta_{2}\right) k h_{k}\right] \sin \left(k \Omega_{0} x\right)\right\}= \\
& \epsilon_{d 2}\left\{\sum_{k}\left[\operatorname{coth}\left(k \Omega_{0} \delta_{2}\right) k g_{k}-\operatorname{csch}\left(k \Omega_{0} \delta_{2}\right) k p_{k}\right] \cos \left(k \Omega_{0} x\right)+\left[\operatorname{coth}\left(k \Omega_{0} \delta_{2}\right) k h_{k}-\operatorname{csch}\left(k \Omega_{0} \delta_{2}\right) k q_{k}\right] \sin \left(k \Omega_{0} x\right)\right\}
\end{aligned}
$$

Similarly, for the coefficients for the $\sin (\cdot)$ terms in Eq. 42, we have:

$$
\epsilon_{d 1}\left[\operatorname{csch}\left(k \Omega_{0} \delta_{1}\right) k b_{k}-\operatorname{coth}\left(k \Omega_{0} \delta_{2}\right) k h_{k}\right]=\epsilon_{d 2}\left[\operatorname{coth}\left(k \Omega_{0} \delta_{2}\right) k h_{k}-\operatorname{csch}\left(k \Omega_{0} \delta_{2}\right) k q_{k}\right]
$$

The tangential components of the electric fields in the dielectric 2 and air at $y=\delta_{1}+\delta_{2}$ must satisfy

$$
E_{a, x}\left(x, \delta_{1}+\delta_{2}\right)=E_{d 2, x}\left(x, \delta_{1}+\delta_{2}\right)
$$

clear that the condition is satisfied automatically. For the normal components at $y=\delta_{1}+\delta_{2}$, we must have: $\epsilon_{0} E_{a, y}=\epsilon_{0} \epsilon_{d 2} E_{d 2, y}$ or $E_{a, y}=\epsilon_{d 2} E_{d 2, y}$. Hence,

$$
\begin{aligned}
\sum_{k}\left[k p_{k} \cos \left(k \Omega_{0} x\right)+k q_{k} \sin \left(k \Omega_{0} x\right)\right]= & \epsilon_{d 2} \cdot\left\{\sum_{k} \operatorname{csch}\left(k \Omega_{0} \delta_{2}\right)\left[k g_{k} \cos \left(k \Omega_{0} x\right)+k h_{k} \sin \left(k \Omega_{0} x\right)\right]-\right. \\
& \sum_{k} \operatorname{coth}\left(k \Omega_{0} \delta_{2}\right)\left[k p_{k} \cos \left(k \Omega_{0} x\right)+k q_{k} \sin \left(k \Omega_{0} x\right)\right]
\end{aligned}
$$

By re-arranging the terms for the $\cos (\cdot)$ and $\sin (\cdot)$ terms in Eq. 46, we arrive at the following expressions for $p_{k}$ and $q_{k}$ :

$$
p_{k}=\frac{\epsilon_{d 2}}{\sinh \left(k \Omega_{0} \delta_{2}\right)+\epsilon_{d 2} \cosh \left(k \Omega_{0} \delta_{2}\right)} g_{k}
$$




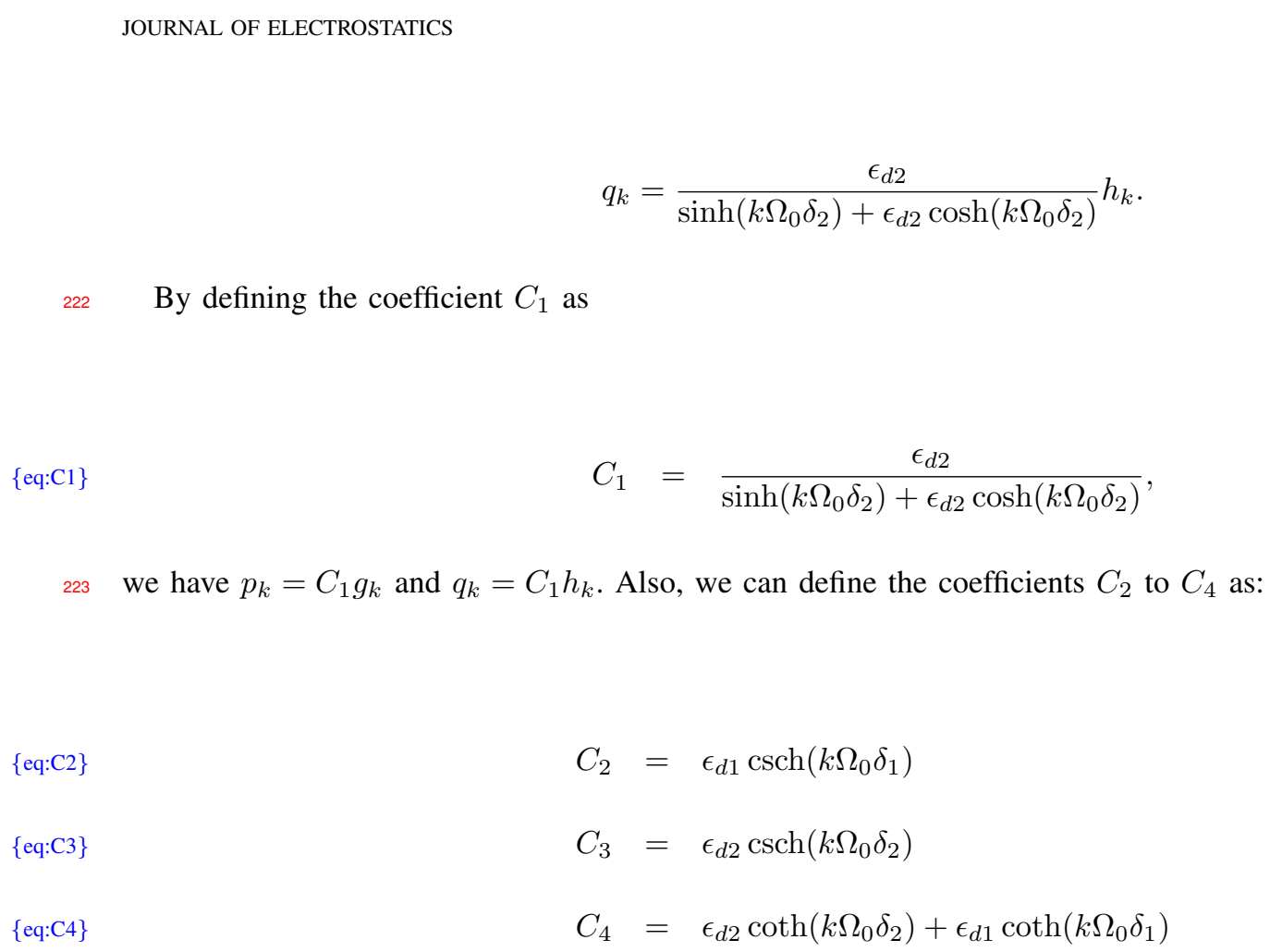$$
q_{k}=\frac{\epsilon_{d 2}}{\sinh \left(k \Omega_{0} \delta_{2}\right)+\epsilon_{d 2} \cosh \left(k \Omega_{0} \delta_{2}\right)} h_{k} .
$$

222

$\{\mathrm{eq}: \mathrm{C} 1\}$

223

we have $p_{k}=C_{1} g_{k}$ and $q_{k}=C_{1} h_{k}$. Also, we can define the coefficients $C_{2}$ to $C_{4}$ as:

$\{$ eq:C2\}

$\{\mathrm{eq}: \mathrm{C} 3\}$

$\{\mathrm{eq}: \mathrm{C} 4\}$

$C_{2}=\epsilon_{d 1} \operatorname{csch}\left(k \Omega_{0} \delta_{1}\right)$

$C_{3}=\epsilon_{d 2} \operatorname{csch}\left(k \Omega_{0} \delta_{2}\right)$

$C_{4}=\epsilon_{d 2} \operatorname{coth}\left(k \Omega_{0} \delta_{2}\right)+\epsilon_{d 1} \operatorname{coth}\left(k \Omega_{0} \delta_{1}\right)$

224

225

34

35

36 as follows:

$$
\begin{aligned}
C_{2} a_{k}+C_{3} p_{k} & =C_{4} g_{k}, \\
C_{2} b_{k}+C_{3} q_{k} & =C_{4} h_{k} .
\end{aligned}
$$

226 227

\{eq:gk\}

\{eq:hk\}

\{eq:pk\}

\{eq:qk\} $q_{k}$, respectively, as:

$$
\begin{aligned}
g_{k} & =\frac{C_{2}}{C_{4}-C_{1} C_{3}} a_{k} \\
h_{k} & =\frac{C_{2}}{C_{4}-C_{1} C_{3}} b_{k} \\
p_{k} & =\frac{C_{1} C_{2}}{C_{4}-C_{1} C_{3}} a_{k} \\
q_{k} & =\frac{C_{1} C_{2}}{C_{4}-C_{1} C_{3}} b_{k}
\end{aligned}
$$

Based on the definitions provided for the coefficients $C_{2}$ to $C_{4}$ in Eqs. 50 to 52 , Eqs. 43 and 44 can be updated

Since the Fourier coefficients $a_{k}$ and $b_{k}$ are known, we have the unknown Fourier coefficients $g_{k}, h_{k}, p_{k}$, and 
By noting that $\Omega_{0}=\frac{2 \pi}{3(w+g)}$, it is obvious that the electric potential and the electric field components discussed above are dependent upon the geometric parameters $w, g, \delta_{1}$, and $\delta_{2}$ and the electrical permittivities of the two dielectric coatings $\epsilon_{d 1}$ and $\epsilon_{d 2}$. In the next section, we have provided expressions based upon the pulse patterns of the three-phase power supply to show how the Fourier coefficients $a_{k}$ and $b_{k}$ are calculated.

\section{NUMERICAl SOlUtion}

\section{${ }_{234}$ A. Verification of Analytical Approach}

In order to verify the closed-form solutions provided in Section 2, the EDS configuration presented in Fig. 1 has been developed in the COMSOL Multiphysics ${ }^{\circledR}$ finite element analysis (FEA) software. We are interested to compare the solutions from the two methods to see which one is more useful in what circumstances. The results have been shown in Fig. 2 for the electric field norm, defined as $|\mathbf{E}|=\left(E_{x}^{2}+E_{y}^{2}\right)^{0.5}$ and the electric potential when the phases $\mathrm{A}, \mathrm{B}$, and $\mathrm{C}$ have the voltages $1 \mathrm{kV}, 0$, and $1 \mathrm{kV}$, respectively. As can be expected and also is shown in Fig. 2, the electric field intensity reaches its maximum in the vicinity of the electrodes tips. Although studying the electric field intensity in the dielectric layers and its dependency upon the geometric as well as operational parameters will provide valuable information on the dielectric breakdown and stress, it is not in the scope of this study. In this paper, we are mainly investigating the electric field distribution on the EDS, where dust particles are deposited and exposed to different attracting and repelling forces.

The electrodes of the EDS are connected to a three-phase power supply that energizes the electrodes with rectangular pulses at $50 \%$ duty cycle. Figure 3 shows these waveforms over a single period $T_{t}$. As can be seen in Fig. 3. there are a total 6 different voltage combinations in which each phase has 0 or $V$ volts. Suppose the voltages of the first, second, and third electrode in one fundamental spatial period $T_{s}$ are denoted as $V_{1}, V_{2}$, and $V_{3}$, respectively, in which they switch between 0 and $V$, as illustrated in Fig. 3 As shown in previous section, the analytical solution is dependent upon the Fourier coefficients $a_{k}$ and $b_{k}$. To obtain these coefficients, we need to have the electric potential at $y=0$ (Fig. 11). The electric potential at the $y=0$ with the assumption of linear voltage change between two adjacent electrodes can be expressed as: 
Surface: Electric field norm $(\mathrm{V} / \mathrm{m})$

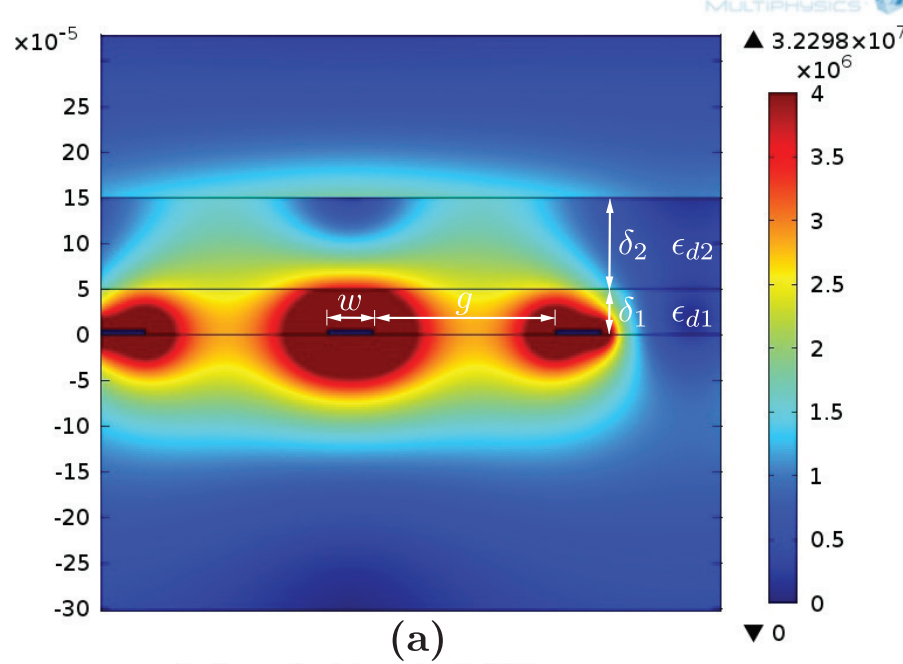

Surface: Electric potential (V)

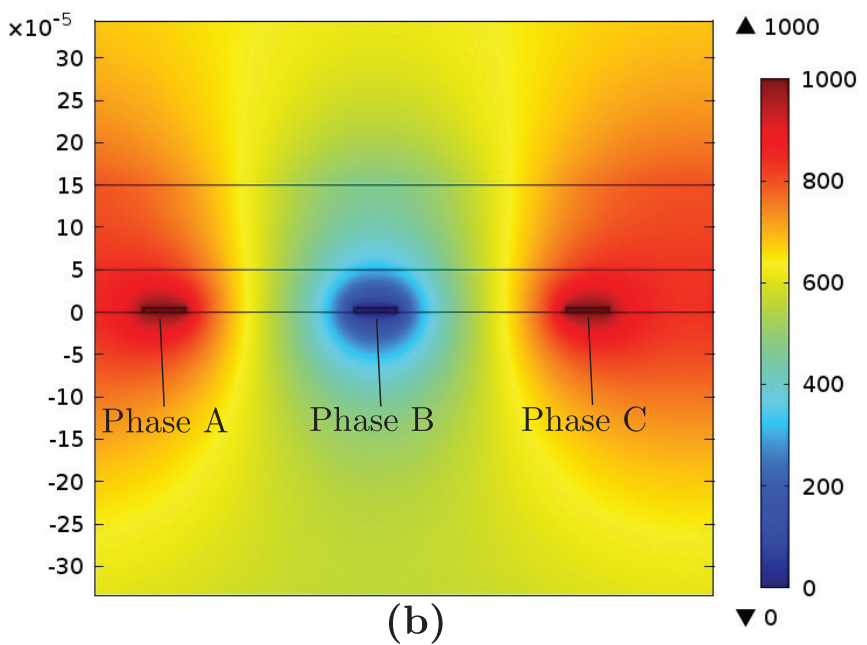

Fig. 2. Numerical simulation results using finite element analysis software for the (a) electric field norm (|E|) and (b) electric potential of the EDS model in one fundamental spatial period $T_{s}$. The unit of the vertical and horizontal axes is meter. The height of the electrodes have been considered to be $5 \mu \mathrm{m}$ and the maximum of the electric field norm reaches $3.2298 \times 10^{7} \mathrm{~V} / \mathrm{m}$. The voltages of phases A, B, and C with Chromium electrodes are $1 \mathrm{kV}, 0$, and $1 \mathrm{kV}$, respectively. The electrode width and inter-electrode spacing are considered to be $w=50$ $\mu \mathrm{m}$ and $g=200 \mu \mathrm{m}$, respectively. The thicknesses of the first and second user-defined dielectric layers are $\delta_{1}=50 \mu \mathrm{m}$ and $\delta_{2}=100 \mu \mathrm{m}$, respectively, and their relative permittivities are $\epsilon_{d 1}=5.14$ and $\epsilon_{d 2}=6.7$. 

expressions for $a_{k}$ and $b_{k}$, respectively, as follows:

$$
\begin{aligned}
a_{k}= & \frac{1}{\pi g \Omega_{0} k^{2}}\left\{\left(V_{2}-V_{1}\right)\left[\cos \left(k \Omega_{0}(w+g)\right)-\cos \left(k \Omega_{0} w\right)\right]+\right. \\
& \left.\left(V_{3}-V_{2}\right)\left[\cos \left(2 k \Omega_{0}(w+g)\right)-\cos \left(k \Omega_{0}(2 w+g)\right)\right]+\left(V_{1}-V_{3}\right)\left[1-\cos \left(k \Omega_{0}(3 w+2 g)\right)\right]\right\} \\
b_{k}= & \frac{1}{\pi g \Omega_{0} k^{2}}\left\{\left(V_{2}-V_{1}\right)\left[\sin \left(k \Omega_{0}(w+g)\right)-\sin \left(k \Omega_{0} w\right)\right]+\right. \\
& \left.\left(V_{3}-V_{2}\right)\left[\sin \left(2 k \Omega_{0}(w+g)\right)-\sin \left(k \Omega_{0}(2 w+g)\right)\right]-\left(V_{1}-V_{3}\right) \sin \left(k \Omega_{0}(3 w+2 g)\right)\right\}
\end{aligned}
$$

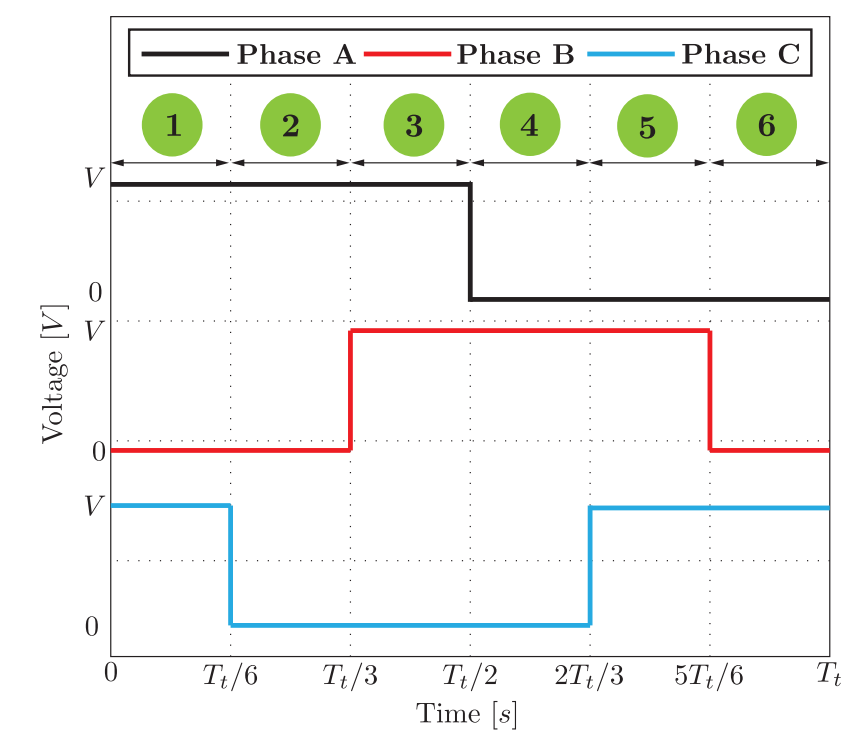

Fig. 3. Three-phase rectangular voltage pulses with $50 \%$ duty cycle in one period $T_{t}$.

The Fourier coefficients $a_{k}$ and $b_{k}$, addressed in Eq. 3, are obtained using the following expressions:

in which $\Omega=k \Omega_{0}$ and $\phi_{d 1}(x, 0)$ is provided in Eq. 59 . By substituting Eq. 59 into Eqs. 60 and 61 , we have the

In order to verify the analytical results obtained in previous section, Fig. 4 has been provided, in which the electric field norm on the EDS surface (i.e. $y=\delta_{1}+\delta_{2}$ ) is calculated from two approaches: analytical (ANA) and finite element analysis (FEA) for six different voltage combinations introduced in Fig. 3. To obtain the electric field 
components from the ANA method, the Fourier coefficients $a_{k}$ and $b_{k}$ were initially calculated for each case of voltage combination from Eqs. 62 and 63 Then, Fourier coefficients $g_{k}, h_{k}, p_{k}$, and $q_{k}$ are calculated according to Eq. 55 to 58 respectively. The expressions to calculate coefficients $C_{1}$ to $C_{4}$ are provided in Eqs. 49 to 52. respectively. The electric field components on the EDS surface (i.e. $y=\delta_{1}+\delta_{2}$ ) can be calculated using the expressions for the electric field distribution in the air as provided in Eqs. 39 and 40 or using the electric field components in the second dielectric coating at $y=\delta_{1}+\delta_{2}$ provided in Eqs. 37 and 38 . As can be seen in Fig. 4 the electric field norm calculated from analytical approach does not fully follow the one provided by FEA method. To examine the accuracy of the analytical solutions, we set the FEA method as the base for comparison and define the parameter "error percentage" as:

$$
\text { Error Percentage }=\frac{\mid \text { FEA }- \text { ANA } \mid}{\text { FEA }} \times 100 \%
$$

The electric field norms provided in Fig. 4 have been compared with each other using Eq. 64 The maximum value of error percentage between the two methods shown in Fig. 4. calculated using Eq. 64 is $4.27 \%, 10.29 \%$, $4.25 \%, 10.44 \%, 4.06 \%, 10.56 \%$, for cases 1 through 6 , respectively. Also, the average of maximum values of error percentages for the six cases is $6.81 \%$. The maximum value of $E_{y}$ component on the EDS surface in cases 2, 4, and 6 is $2.09 \times 10^{6} \mathrm{~V} / \mathrm{m}$ while the maximum of $E_{y}$ is $1.47 \times 10^{6}$ in cases 1,3 , and 5. The $E_{x}$ component reaches its maximum value of $1.88 \times 10^{6} \mathrm{~V} / \mathrm{m}$ in all six cases. The maximum and minimum values of electric field norm in cases 1,3 , and 5 are $1.41 \times 10^{6}$ and $2.04 \times 10^{6} \mathrm{~V} / \mathrm{m}$, respectively. In cases 2,4 , and 6 , the $|\mathbf{E}|$ attains its maximum and minimum values of $2.12 \times 10^{6}$ and $1.33 \times 10^{6} \mathrm{~V} / \mathrm{m}$, respectively.

It should be pointed out that the difference between the results from the two methods, as can be observed in Fig. 4 is attributable to the fact that the voltage change between adjacent electrodes has been considered to be linear in the analytical approach while FEA method provides a more complex voltage profile. Figure 5 depicts the electric potential at $y=0$, where the electrodes are located, for the two ANA and FEA methods. In Fig. 5, when one of the two adjacent electrodes is at zero electric potential and the other at 1000 volts, linear voltage profile used in the analytical calculations is merely an approximation of the profile that FEA method provides. The most significant deviation between the two voltage profiles occurs when adjacent electrodes are both at zero potential or at $1 \mathrm{kV}$. For instance, when both phases $\mathrm{A}$ and $\mathrm{B}$ are at $1 \mathrm{kV}$, Fig. 5 case 3, FEA method provides a voltage profile that decreases down to 905 volts while the ANA method maintains the voltage at 1000 volts between the electrodes. Therefore, linear approximation introduces an error up to $10.49 \%$ in the electric potential in this case. 
Similarly, in case 6 of Fig. 5 when both A and B phases are at zero electric potential, the voltage profile obtained from FEA method experiences an increase up to 85.5 volts along the $x$ axis while linear approximation assumes the electric potential remains at 0 between the phases. The nonlinear behavior of the electric potential between two adjacent equipotential electrodes in the two aforementioned cases and other similar cases, is attributable to the neighboring electrodes. In case 3 of Fig. 5, for instance, the electric potential between phases A and B which are at $1 \mathrm{kV}$, is affected by the phase $\mathrm{C}$ in current and previous spatial periods that are at zero electric potential. Along similar lines, in case 6 of Fig. 5. voltage profile between phases A and B, which are maintained at zero potential, is affected by the presence of phase $\mathrm{C}$ electrodes of previous and current spatial period. Analytical models have been derived for the electric potential between two cylindrical conductors, like [16] or two-wire transmission line [17], 
(1)

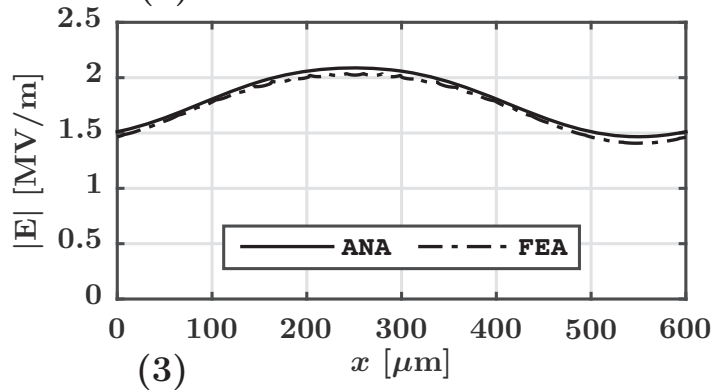

(3)
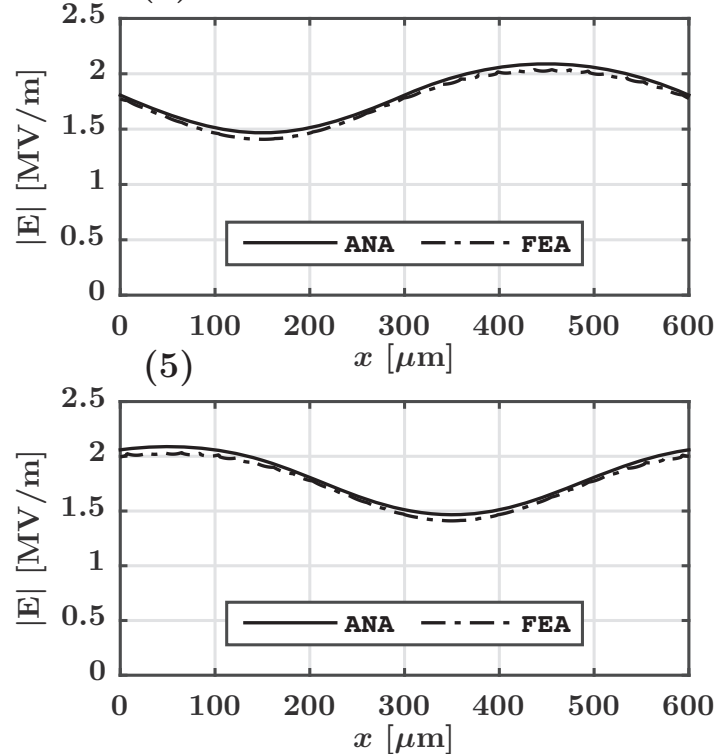

$(2)$
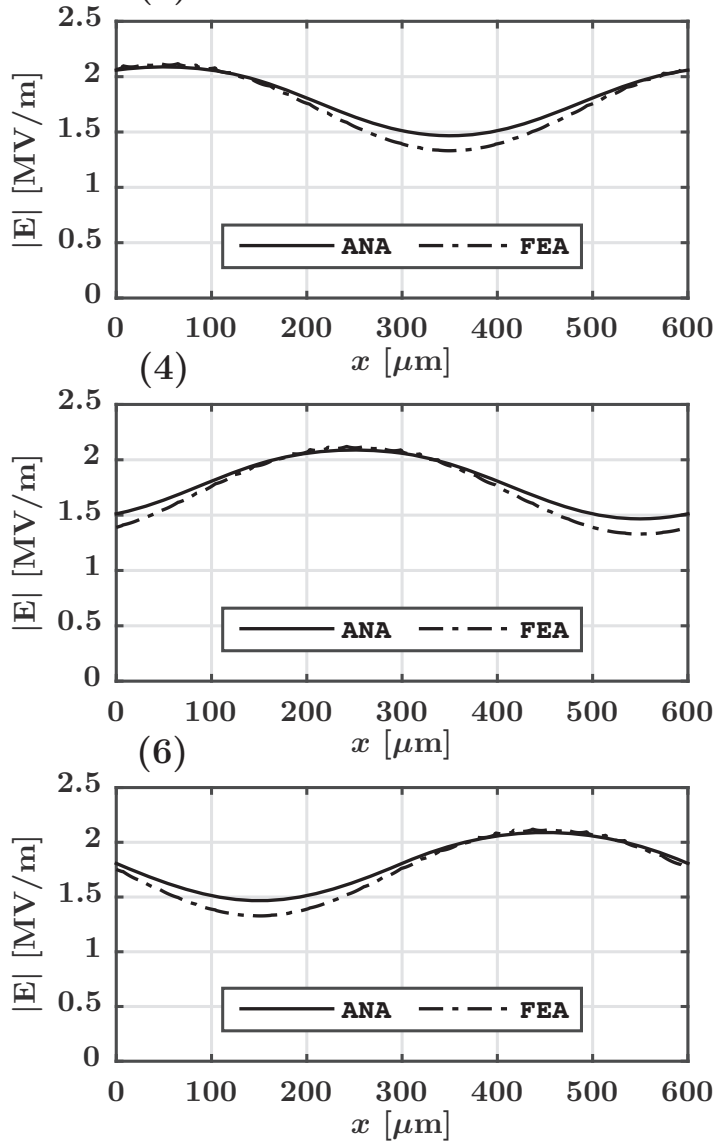

Fig. 4. Comparison of analytical (ANA) results with numerical simulations using finite element analysis (FEA) software for $w=g=100 \mu \mathrm{m}$ in one fundamental spatial period $T_{s}=600 \mu \mathrm{m}$ for six different voltage combinations for phases A, B, and C. The magnitude of the electric field (unit: $[\mathrm{MV} / \mathrm{m}]$ ) on the EDS surface (i.e. $y=\delta_{1}+\delta_{2}$ ) is illustrated vs. $x$. The positions of the first, second, and third electrodes are at $[0,100 \mu \mathrm{m}],[200 \mu \mathrm{m}, 300 \mu \mathrm{m}]$, and [400 $\mu \mathrm{m}, 500 \mu \mathrm{m}]$, respectively. The voltages of phases $(A, B, C)$ for the cases (1) to $(6)$ are respectively as follows: $(V, 0, V),(V, 0,0),(V, V, 0),(0, V, 0),(0, V, V)$, and $(0,0, V)$, in which $V=1000$ volts. The thicknesses of the first and second dielectric coatings are 50 and $100 \mu \mathrm{m}$, respectively, and their relative permittivities are 5.14 and 6.7 .

particles. As analytically provided in the preceding section, the electric field components in the dielectric materials and air are dependent upon the geometric parameters and relative permittivities of two transparent dielectric layers $\epsilon_{d 1}$ and $\epsilon_{d 2}$. It is worth to study how the electric field distribution on the EDS surface, where the dust particles are deposited, behaves as a function of the dielectric coating thickness. For this purpose, we have run the FEA software for different values of the thickness of the first dielectric coating $\delta_{1}$ to see its impact on the electric field distribution on the EDS surface. Figure 6 shows the simulation results of the normal electric field component $E_{y}$ in one fundamental spatial period on the EDS surface when the electrode width and inter-electrode spacing are 50 and $700 \mu \mathrm{m}$, respectively, for various values of the $\delta_{1}$ in the range of $25 \mu \mathrm{m}$ to $140 \mu \mathrm{m}$, while the other parameters are fixed. Although several values for $\delta_{1}$ in the aforementioned range were considered in the simulation study, only 
(1)

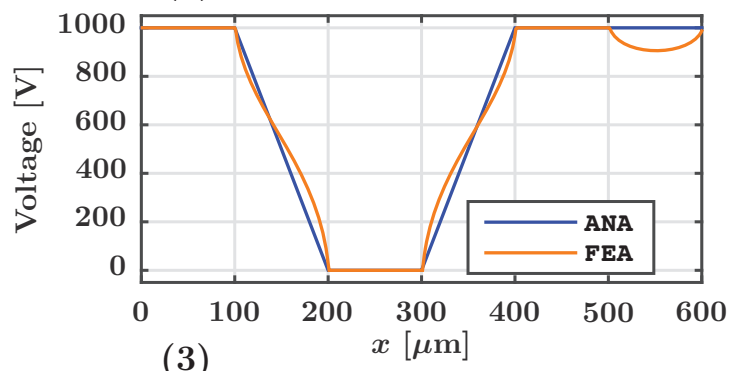

(3)

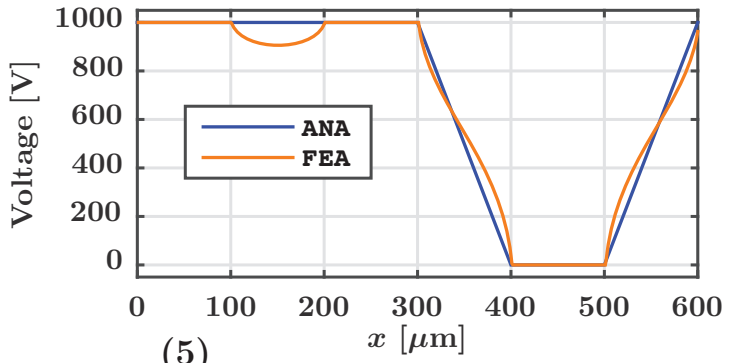

(5)

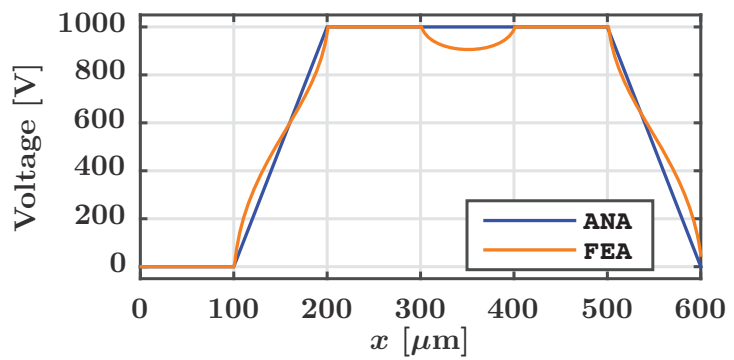

(2)

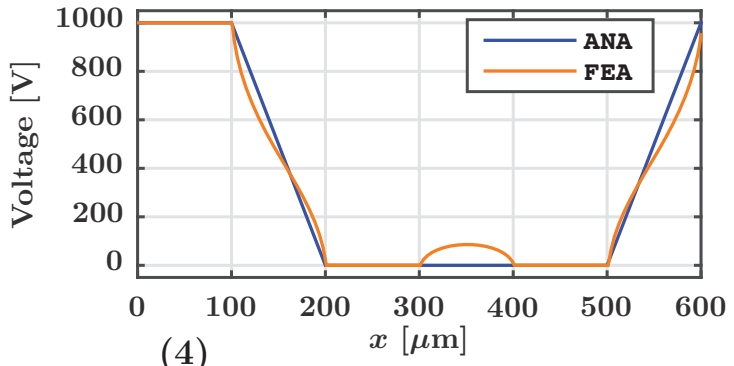

(4)
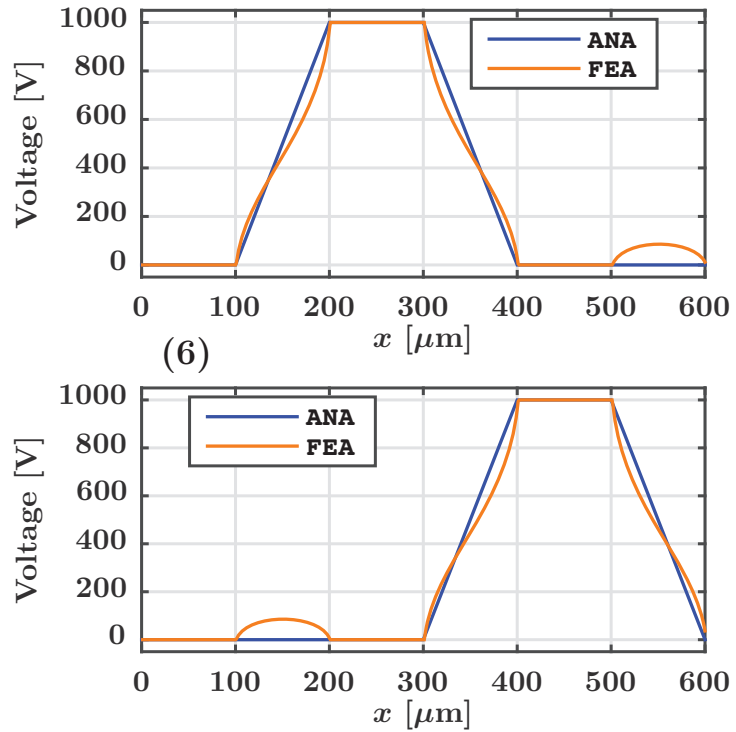

Fig. 5. Comparison of the voltage profiles used in the analytical (ANA) approach and computed with numerical simulations using finite element analysis (FEA) software for $w=g=100 \mu \mathrm{m}$ in one fundamental spatial period $T_{s}=600 \mu \mathrm{m}$ for six different voltage combinations for phases $\mathrm{A}, \mathrm{B}$, and $\mathrm{C}$. The electric potential at $y=0$, where the electrodes are located is shown vs. $x$. The positions of the first, second, and third electrodes are at $[0,100 \mu \mathrm{m}],[200 \mu \mathrm{m}, 300 \mu \mathrm{m}]$, and [400 $\mu \mathrm{m}, 500 \mu \mathrm{m}]$, respectively. The rest of the parameters are identical to Fig. 4

. 377age profile\} 38

39

40

41

42

43

44

45

46

47

48

49

50

51

52

53

54

55

56

57

58

59

60

61

62

63

64

65

four of them are illustrated in Fig. 6 for the sake of readability of the figure. As can be observed in Fig. 6, the $E_{y}$ experiences a significant change when $\delta_{1}$ changes from $50 \mu \mathrm{m}$ to $63 \mu \mathrm{m}$, while when $\delta_{1}$ increases from $63 \mu \mathrm{m}$ to $125 \mu \mathrm{m}$, the $E_{y}$ does not change drastically. The $\delta_{1}=63 \mu \mathrm{m}$ provides the maximum of the $E_{y}$ in the positive direction in all of the the six cases of the voltage combinations.

As mentioned earlier, the Coulomb force is the predominant force in dust particle removal process. Although the motion of dust particles in $x$ and $y$ - direction due to exerted forces are both important in detailed modeling of the particle trajectories, we pay significant attention to the vertical motion of the particle and the balance of repelling and attracting forces. In other words, we endeavor to ensure at each given point on the EDS surface, we maintain the required amount of electric field component in the positive $y$-direction to strengthen the Coulomb force among other forces, assuming the particle is positively charged. When the dust particle is sitting on the EDS surface, the 
gravity and adhesion force are the attracting ones that push the particle into the EDS surface. The adhesion force is the summation of van der Waals and capillary forces [18]. As can be noticed in Fig. 6, the normal component

$(1)$

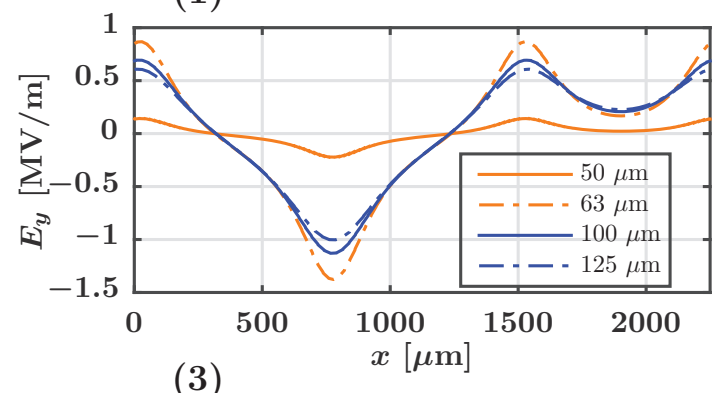

(3)

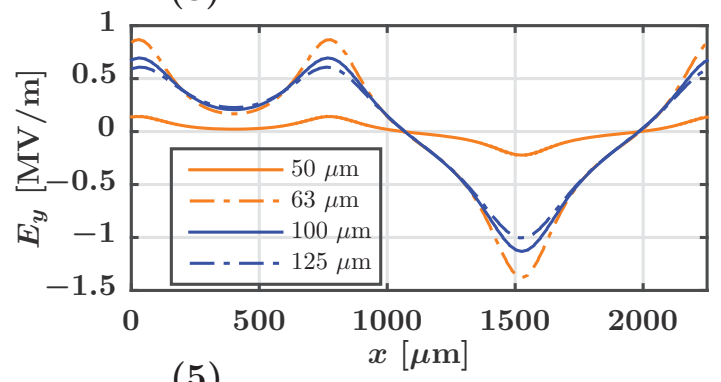

$(5)$

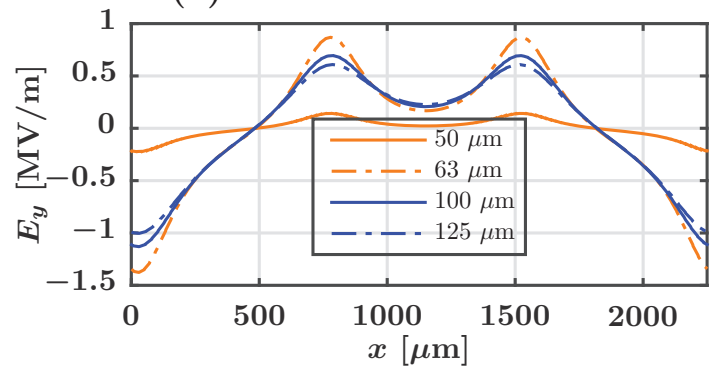

$(2)$

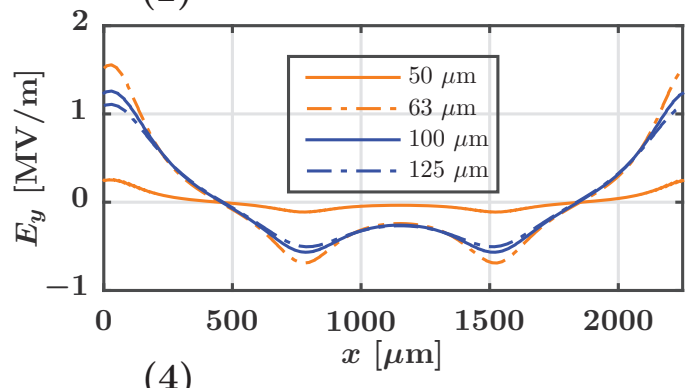

$(4)$

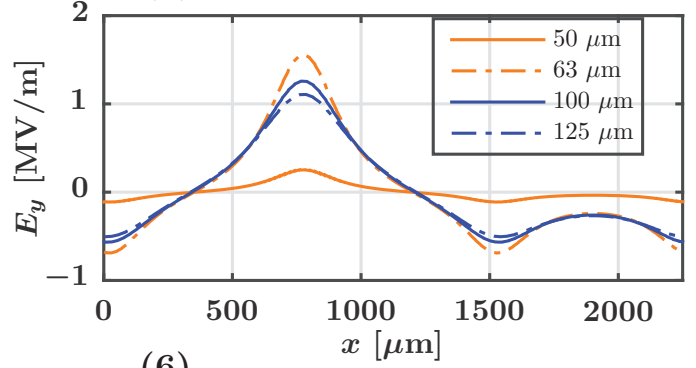

$(6)$

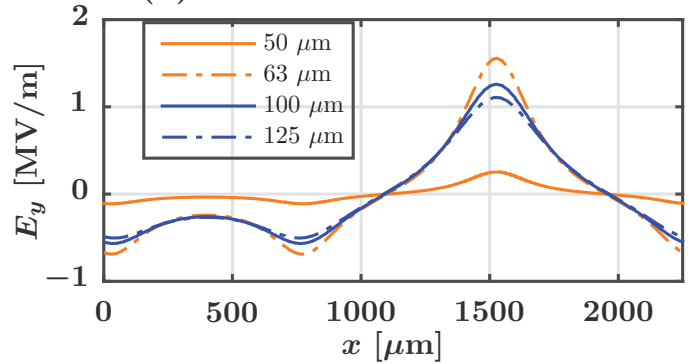

Fig. 6. The comparison between the $E_{y}$ (unit: [MV/m]) components on the EDS surface in one fundamental spatial period for different values of the thickness of the first dielectric coating $\delta_{1}$. The results obtained from the COMSOL Multiphysics software. The positions of the first, second, and third electrodes are at $[0,50 \mu \mathrm{m}],[750 \mu \mathrm{m}, 800 \mu \mathrm{m}]$, and $[1500 \mu \mathrm{m}, 1550 \mu \mathrm{m}]$, respectively, and the fundamental spatial period is $2250 \mu \mathrm{m}$. The voltages of phases $(\mathrm{A}, \mathrm{B}, \mathrm{C})$ for the cases (1) to (6) are respectively as follows: $(V, 0, V),(V, 0,0),(V, V, 0),(0, V, 0),(0, V, V)$, and $(0,0, V)$, in which $V=1000$ volts. The thickness of the second dielectric coating is fixed at $100 \mu \mathrm{m}$ and the relative permittivities of the first and second dielectric layers are 5.14 and 6.7 , respectively. 


\section{A. Geometry}

Figure 7 shows the second EDS configuration considered in this study. The configuration depicted in Fig. 7 is a viable candidate for dust removal from solar mirror in CSP application. As depicted in Fig. 7, EDS consists of three layers of dielectric coatings in which the electrodes are printed on the lower side of the third dielectric layer. Similar to Fig. 1. the electrode width and inter-electrode spacing are denoted as $w$ and $g$, respectively. The thicknesses of transparent dielectric layers 1 to 3 are denoted as $\delta_{1}, \delta_{2}$, and $\delta_{3}$, respectively, and their relative permittivities highlighted as $\epsilon_{d 1}, \epsilon_{d 2}$, and $\epsilon_{d 3}$. In our laboratory work, the dielectric layers 1, 2, and 3 are considered to be acrylic, optically clear adhesive (OCA) [19], and Corning ${ }^{\circledR}$ Willow ${ }^{\circledR}$ Glass [20], respectively. The metal layer is usually a thin layer, in the order of 1-2 $\mu \mathrm{m}$ of Aluminum or Silver. The dielectric constants of acrylic, OCA, and Corning ${ }^{\circledR}$ Willow ${ }^{\circledR}$ Glass have been considered to be $3.5,5.14$, and 5.5, respectively, throughout the FEA study.

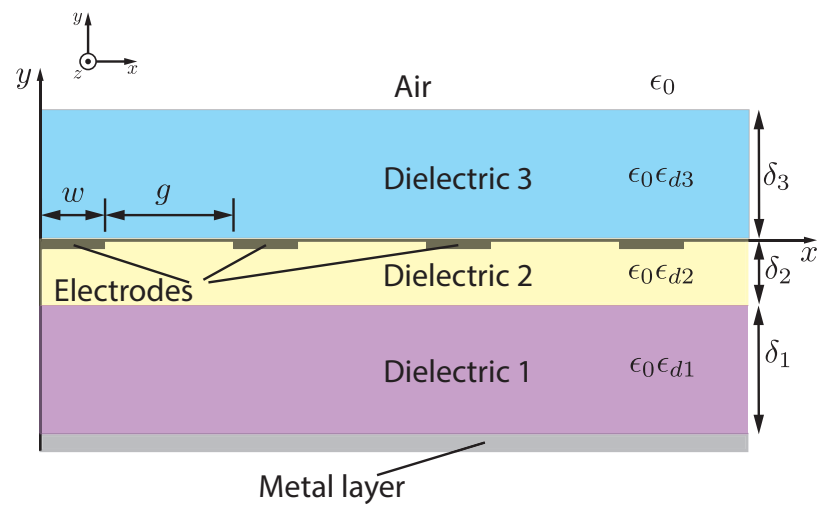

Fig. 7. The second EDS configuration, in which the electrodes are printed on the lower side of dielectric layer 3.

\section{B. Numerical Results}

The EDS configuration has been developed in the COMSOL Multiphysics simulation software and the results for the electric potential and electric field are shown in Figure 8, in which the electrode width and inter-electrode spacing are set to be 75 and $300 \mu \mathrm{m}$, respectively. In Fig. 8, the maximum of $|\mathbf{E}|, E_{y}$, and $E_{x}$ reach $2.41 \times 10^{6}$, $4.7 \times 10^{5}$, and $2.38 \times 10^{6} \mathrm{~V} / \mathrm{m}$, respectively, on the EDS surface in the fundamental spatial period of $1125 \mu \mathrm{m}$. The height of the electrodes and the thickness of underlying of metal layer are much smaller than the dimensions of other parameters like electrode width and the thickness of dielectric layers: they have been considered to be $2 \mu \mathrm{m}$. In addition, we are interested to study the electric field distribution on the EDS more thoroughly rather 
than the electric field intensity inside the dielectric layers or in the vicinity of the electrodes. Hence, by defining three different mesh patterns: (1) extremely coarse for conductors, (2) extra fine for the dielectric layers, and (3) extremely fine on the EDS surface, we were able to reduce the mesh generation time significantly yet having accurate computations.

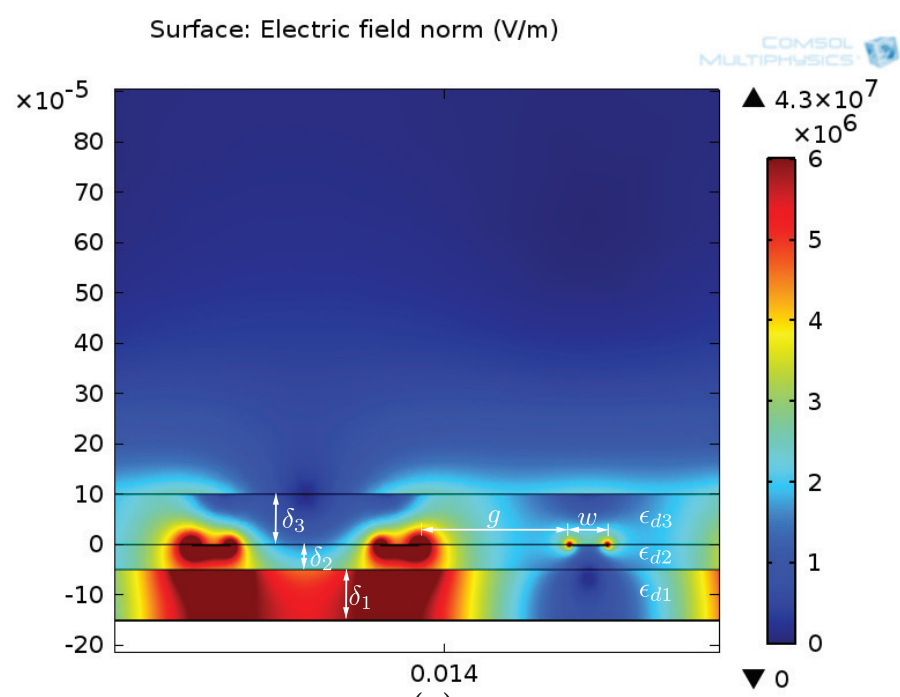

(a)

Surface: Electric potential (V)

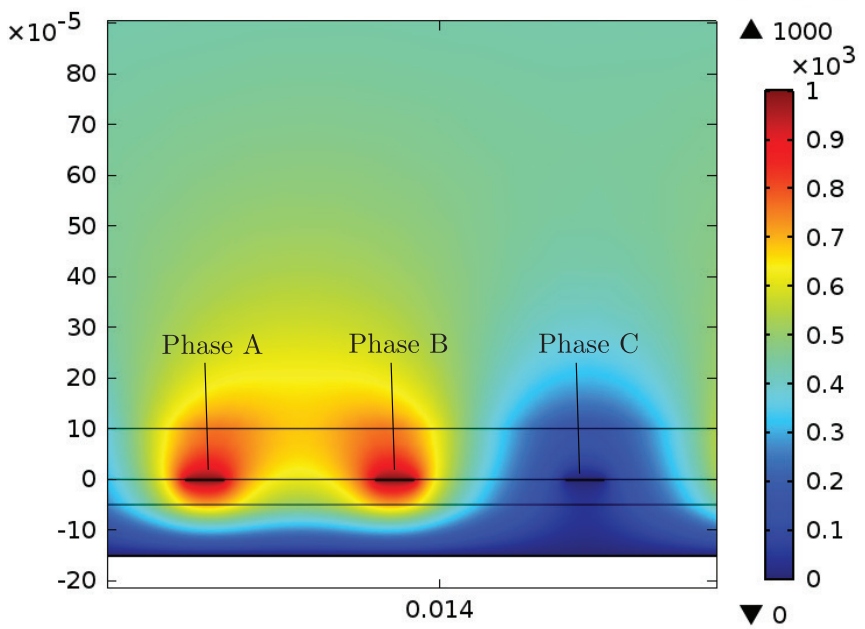

(b)

Fig. 8. Numerical simulation results using finite element analysis software for the (a) electric field norm $|\mathbf{E}|$ (unit: V/m) and (b) electric potential (unit: V) of the second EDS configuration model in one fundamental spatial period $T_{s}$. The unit of the vertical and horizontal axes is meter. The height of the electrodes and the thickness of the metal layer have been considered both to be $2 \mu \mathrm{m}$ and the maximum of the electric field norm reaches $1.8 \times 10^{6} \mathrm{~V} / \mathrm{m}$ on the EDS surface. The voltages of phases $\mathrm{A}, \mathrm{B}$, and $\mathrm{C}$ are $1 \mathrm{kV}, 1 \mathrm{kV}$, and 0 , respectively. The electrode width and inter-electrode spacing are considered to be $w=75 \mu \mathrm{m}$ and $g=300 \mu \mathrm{m}$, respectively. The thicknesses of the first, second, and third user-defined dielectric layers are $\delta_{1}=100 \mu \mathrm{m}, \delta_{2}=50 \mu \mathrm{m}, \delta_{3}=100 \mu \mathrm{m}$, respectively, and their relative permittivities are $\epsilon_{d 1}=3.5$, $\epsilon_{d 2}=5.14$, and $\epsilon_{d 3}=5.5$.

Thus far, we have analyzed the electric field distribution in two EDS configurations. In order to compare these two configuration to realize which one of these geometries performs better regarding dust removal, we consider 
the normal electric field component $E_{y}$ on the EDS surface in one fundamental spatial period, while it is tried to fix all the other parameters. Figure 9 shows the comparison between the $E_{y}$ components for six different voltage combinations in one fundamental spatial period and on the EDS surface. For both configurations, the electrode width and inter-electrode spacing are considered to be 50 and $700 \mu \mathrm{m}$, respectively. For the first EDS configuration (Fig. 1), the thicknesses of the first and second dielectric layers are 50 and $100 \mu \mathrm{m}$, respectively, with relative permittivities of 5.14 and 6.7. For the second EDS configuration (Fig. 7), the thickness of the first, second, and third transparent dielectric layers are 100,50 , and $100 \mu \mathrm{m}$, respectively, with relative permittivities of 3.5, 5.14, and 5.5. The mentioned dimensions are typical for EDS.

As can been seen in Fig. 9, the $E_{y}$ in both configurations attain their extrema (maxima and minima) at identical points along the $x$-direction in one fundamental spatial period. However, the second EDS configuration provides higher values for $E_{y}$ at the maxima for all the cases. The percentage of increase in the $E_{y}$ of second configuration at the maxima for the cases (1) through (6) are $63.86 \%, 16.66 \%, 66.36 \%, 15.41 \%, 62.98 \%$, and $15.32 \%$, respectively, compared to the first configuration. Also, it is interesting to note that what percent of one fundamental spatial period the values $E_{y}$ of second configuration are greater than that of first configuration. In $40.98 \%, 56.12 \%$, $41.16 \%, 55.68 \%, 40.49 \%$, and $40.98 \%$ of the fundamental spatial period, the second EDS configuration yields higher values for $E_{y}$ than the first configuration for the cases (1) through (6), respectively.

\section{CONCLUSION}

Electric field distribution model is an essential part in performance analysis of the electrodynamic screen (EDS) to achieve high dust removal rates. The ultimate goal is to ensure that at each given point on the EDS surface, the summation of repelling forces are greater than the attracting ones in the balance of exerted forces on the particle. In this paper, we provided the analytical solutions for the electric potential and electric field distribution in an EDS configuration with two stacked layers of transparent dielectric coatings. The dependency of the solutions on various design parameters as well as electrical properties of the dielectric materials was emphasized. Corroboration of the analytical solutions using the developed EDS model in the FEA software showed that the average of maximum error percentages between the solutions is less than $7 \%$ for six voltage combinations. By providing more accurate expressions for the voltage profile between adjacent electrodes in the analytical method, the error percentage can be reduced much further. The optimal value for the thickness of one of the dielectric layers in order to maximize the normal electric field component on the EDS surface was reported. Furthermore, the electric field analysis for the second EDS configuration was performed using FEA software. In order to evaluate the two configurations, 
(1)

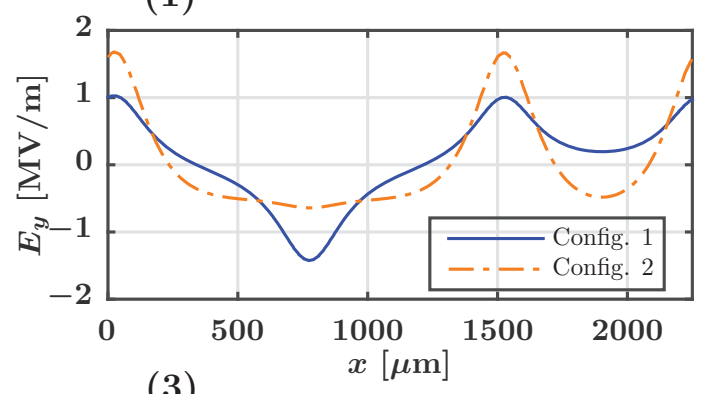

(3)

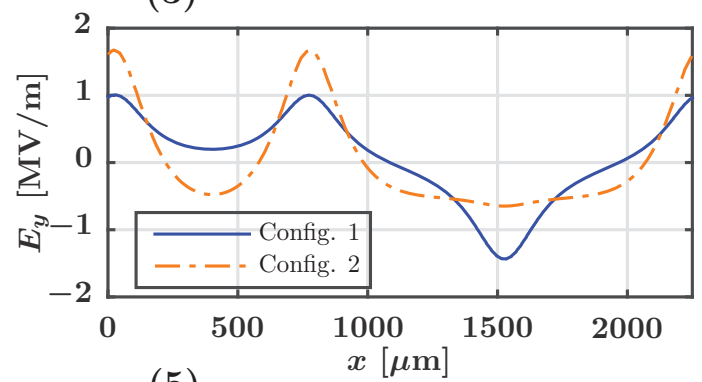

(5)

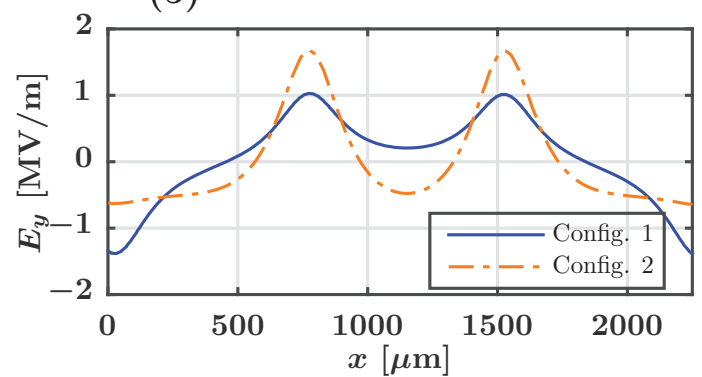

(2)

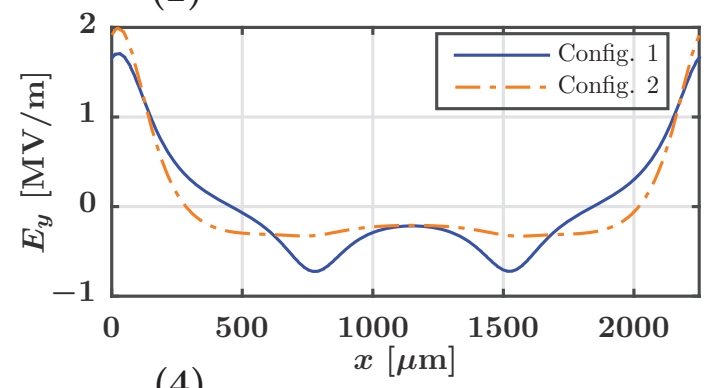

(4)

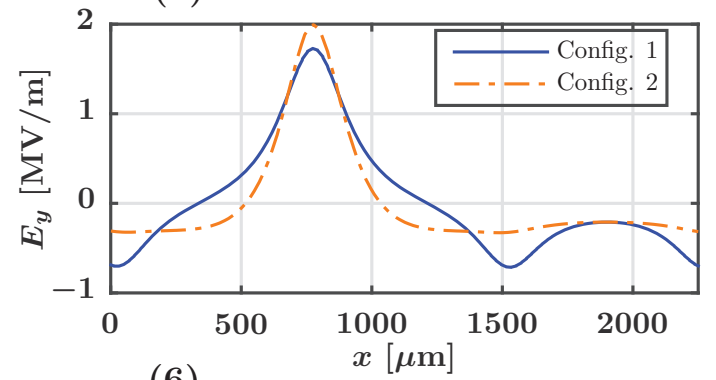

(6)

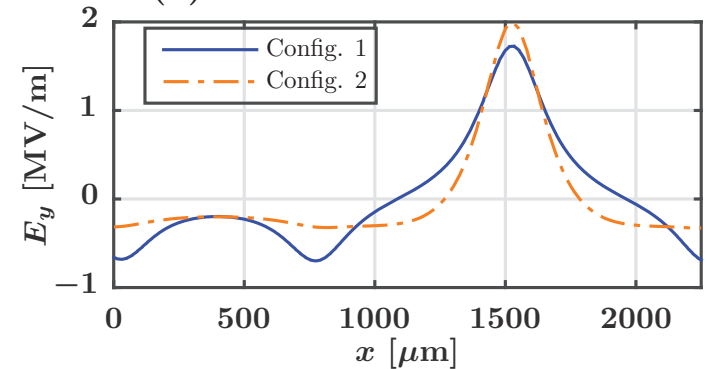

Fig. 9. The comparison between the $E_{y}$ (unit: $[\mathrm{MV} / \mathrm{m}]$ ) components on the EDS surface in one fundamental spatial period for the two EDS configurations. The electrode width, inter-electrode spacing, and fundamental spatial period are 50, 700 , and $2250 \mu \mathrm{m}$, respectively. The positions of the first, second, and third electrodes are at [0,50 $\mu \mathrm{m}]$, [750 $\mu \mathrm{m}, 800 \mu \mathrm{m}]$, and [1500 $\mu \mathrm{m}, 1550 \mu \mathrm{m}]$, respectively. The voltages of phases $(\mathrm{A}, \mathrm{B}, \mathrm{C})$ for the cases (1) to (6) are respectively as follows: $(V, 0, V),(V, 0,0),(V, V, 0),(0, V, 0),(0, V, V)$, and $(0,0, V)$, where $V=1000$ volts.

the normal electric field components on the EDS surfaces in two configurations was compared. It was found that when the design as well as operational parameters are kept identical, there is no significant distinction between two configurations. More criteria can be considered for further evaluation of the EDS configurations. The results of this paper are beneficial in detailed dust particle trajectory modeling and optimization procedure of the EDS for higher dust removal rates. 
Fourier coefficients

locally defined electric potential function, V

inter-electrode spacing, $\mathrm{m}$

Fourier coefficients

index of summation

Fourier coefficients

fundamental spatial period, $\mathrm{m}$

time period, $\mathrm{s}$

electric potential at $y=\delta_{1}, \mathrm{~V}$

electric potential at $y=0, \mathrm{~V}$

electrode width, $\mathrm{m}$

electric potential at $y=\delta_{1}+\delta_{2}, \mathrm{~V}$

unit vector in $x$ direction, $\mathrm{m}$

unit vector in $y$ direction, $\mathrm{m}$

electric field vector, $\mathrm{V} / \mathrm{m}$

tangential component of the electric field, $\mathrm{V} / \mathrm{m}$

normal component of the electric field, $\mathrm{V} / \mathrm{m}$

Greek symbols

thickness of the transparent dielectric layer, $\mathrm{m}$

relative permittivity of the dielectric coating

permittivity of free space, $8.854 \times 10^{-12} \mathrm{~F} / \mathrm{m}$

electric potential, $\mathrm{V}$

spatial angular frequency, $\mathrm{rad} / \mathrm{m}$

Subscript

air

dielectric 1

dielectric 2

dielectric 3 


\section{REFERENCES}

[1] A. Sayyah, M. N. Horenstein, and M. K. Mazumder, "Energy yield loss caused by dust deposition on photovoltaic panels," Solar Energy, vol. 107, no. 0, pp. 576 - 604, 2014. [Online]. Available: http://www.sciencedirect.com/science/article/pii/S0038092X1400259X

[2] E. Asl-Soleimani, S. Farhangi, and M. S. Zabihi, "The effect of tilt angle, air pollution on performance of photovoltaic systems in Tehran," Renewable Energy, vol. 24, no. 3-4, pp. 459-468, November 2001.

[3] L. Liqun, L. Zhiqi, and S. Z. L. Chunxia, "Degraded output characteristic at atmospheric air pollution and economy analysis of PV power system: A case study," PRZEGLAD ELEKTROTECHNICZNY (Electrical Review), vol. 88, no. 9A, pp. 281-284, 2012.

[4] A. Sayyah, M. Horenstein, and M. Mazumder, "Mitigation of soiling losses in concentrating solar collectors," in Photovoltaic Specialists Conference (PVSC), 2013 IEEE 39th, Tampa, FL, June 2013, pp. 0480-0485.

[5] M. Anderson, A. Grandy, J. Hastie, A. Sweezey, R. Ranky, and C. Mavroidis, "Robotic device for cleaning photovoltaic panel arrays," in 12th International Conference on Climbing and Walking Robots and the Support Technologies for Mobile Machines, Istanbul, Turkey, 9-11 September 2009, pp. 1-11.

[6] F. Tatom, V. Srepel, R. Johnson, N. Contaxes, J. Adams, H. Seaman, and B. Cline, "Lunar dust degradation effects and removal/prevention concepts,” National Aeronautics and Space Administration (NASA), Tech. Rep. TR-792-7-207A, 1967.

[7] S. Masuda, K. Fujibayashi, K. Ishida, and H. Inaba, "Confinement and transportation of charged aerosol clouds via electric curtain," Electrical Engineering in Japan, vol. 92, no. 1, pp. 43-52, 1972.

[8] S. Masuda and Y. Matsumoto, "Contact-type electric curtain for electrodynamical control of charged dust particles," in 2nd International Conference on Static Electricity, no. 72, Frankfurt, Germany, March 1973, pp. 1370-1409.

[9] M. Mazumder, M. Zahn, R. Sharma, J. Zhang, C. Calle, C. Immer, and N. Mardesich, "Development of self-cleaning transparent dust shields using low-power electrodynamic fields for solar panels on Mars," in ESA/IEEE-IAS/IEJ/SFE Joint Conference on Electrostatics, Berkeley, CA, 6-9 June 2006, pp. 177-204.

[10] R. Sharma, C. A. Wyatt, J. Zhang, C. I. Calle, N. Mardesich, and M. K. Mazumder, "Experimental evaluation and analysis of electrodynamic screen as dust mitigation technology for future Mars missions," IEEE Transactions on Industry Applications, vol. 45, no. 2, pp. 591-596, 2009.

[11] C. I. Calle, C. R. Buhler, J. L. McFall, and S. J. Snyder, "Particle removal by electrostatic and dielectrophoretic forces for dust control during lunar exploration missions," J. Electrostatics, vol. 67, no. 2-3, pp. 89-92, 2009.

[12] C. Calle, C. Buhler, M. Johansen, M. Hogue, and S. Snyder, "Active dust control and mitigation technology for lunar and Martian exploration," Acta Astronautica, vol. 69, no. 11 - 12, pp. 1082 - 1088, 2011. [Online]. Available: http: //www.sciencedirect.com/science/article/pii/S0094576511001883

[13] M. K. Mazumder, M. N. Horenstein, J. W. Stark, P. Girouard, R. Sumner, B. Henderson, O. Sadder, I. Hidetaka, A. S. Biris, and R. Sharma, "Characterization of electrodynamic screen performance for dust removal from solar panels and solar hydrogen generators," IEEE Transactions on Industry Applications, vol. 49, no. 4, pp. 1793-1800, 2013.

[14] H. Kawamoto and T. Shibata, "Electrostatic cleaning system for removal of sand from solar panels," in 39th IEEE Photovoltaic Specialists Conference (PVSC), Tampa, FL, 16-21 June 2013.

[15] M. N. Horenstein, M. K. Mazumder, and R. C. Sumner, "Predicting particle trajectories on an electrodynamic screen - theory and experiment," Journal of Electrostatics, vol. 71, no. 3, pp. 185-188, 2013.

[16] K. Engelen, P. Jacqmaer, and J. Driesen, "Electric and magnetic fields of two infinitely long parallel cylindrical conductors carrying a dc current," The European Physical Journal Applied Physics, vol. 64, no. 02, p. 24515, 2013.

[17] S. J. Orfanidis, Electromagnetic waves and antennas. Rutgers University New Brunswick, NJ, 2014.

[18] S. You and M. P. Wan, "Mathematical models for the van der Waals force and capillary force between a rough particle and surface," Langmuir, vol. 29, no. 29, pp. 9104-9117, 2013.

[19] (2014) 3M 8146-2 Optically Clear Adhesive. [Online]. Available: http://solutions.3m.com/wps/portal/3M/en_US/Electronics_ NA/Electronics/Products/Product_Catalog/ /3M-Optically-Clear-Adhesive-8146-X?N=4294308054+5153906\&Nr=AND(hrcy_id\% 3A5RXVRVKXW2gs_TGHXDLSLVP_N2RL3FHWVK_GPD0K8BC31gv)\&rt=d\#nonjstabSupport

[20] (2014) Corning ${ }^{\circledR}$ Willow ${ }^{\circledR}$ Glass fact sheet. [Online]. Available: http://www.corning.com/WorkArea/downloadasset.aspx?id=51335 